\title{
A SELECTION THEOREM FOR TOPOLOGICAL CONVEX STRUCTURES
}

\author{
M. VAN DE VEL
}

\begin{abstract}
A continuous selection theorem has been obtained for multivalued functions, the values of which are convex sets of certain synthetic convex structures. Applications are given related with superextensions, (semi)lattices, spaces of order arcs, trees, Whitney levels in hyperspaces, and geometric topology. Applications to traditional convexity in vector spaces involve Beer's approximation theorem and a fixed point theorem of Dugundji-Granas. Some other applications (a.o. an invariant arc theorem) appear elsewhere.
\end{abstract}

\section{INTRODUCTION}

Continuous selections of convex-valued functions were studied for the first time by E. Michael in his by now classical paper [32]. Shortly afterwards, he replaced convexity by a condition of equi-homotopy triviality in the sense of $L C^{*}$ and $C^{*},[33,34]$. These results require a finite dimensional domain, and an example of Pixley [43, Theorem 1.1] shows that this restriction is essential. To cover up with the infinite-dimensional case, Michael introduced a notion of generalized convexity, based on "synthetic convex combinations", and he derived a selection theorem for it, [35]. In recent years, modifications of this result have been obtained by Curtis [10] and by Pasicki [41].

The assumptions of the Michael-Curtis and Pasicki Theorems are a compromise between convexity and equi- $L C$ properties, and their convex structures are intimately connected with metric spaces. During the last decades, a quite different viewpoint on synthetic convexity has been developed, which is independent of topology. One considers a family of subsets of a given set $X$, regarded to be the convex sets, subject to the following conditions.

CONV 1. $\varnothing, X$ are convex.

CONV 2. Intersection of a family of convex sets is convex.

CONV 3. Union of an up-directed family of convex sets is convex.

This framework is a natural one in which to formulate familiar convexity concepts, such as convex hull, polytope, half-space, etc. See $\S \S 1,2$. Many papers in the area concentrate on combinatorial problems; see [17, $\S 6$ or 12] for a

Received by the editors August 1, 1990.

1980 Mathematics Subject Classification (1985 Revision). Primary 52A01, 54C65, 54H99; Secondary 06F30, 46A05, 46A55, 54B20, 54F35, 54F45, 54H25.

Key words and phrases. Absolute retract, compact (semi)lattice, continuous selection, convex hyperspace, convex system, Kakutani separation property, LSC multifunction, metric convex structure, space of order arcs, tree, uniform convex structure, Whitney map. 
survey. Topological problems are dealt with in $[22,23]$, and in most of the author's cited papers. A monograph on set-theoretic and topological convexity is in preparation; see [60].

In the presence of an additional topology, a natural question is whether continuous selection theory survives in this context. The main result of this paper is an affirmative answer under a few reasonable and aesthetical conditions:

(i) All convex sets are connected.

(ii) All polytopes are compact.

(iii) Disjoint convex sets are in complementary half-spaces (Kukutani Separation Property).

(iv) The hull operation is uniformly continuous relative to a metric which generates the topology.

(A condition, comparable with (iv), appears in Pasicki's paper.) The entire $\S 3$ below is devoted to a description of examples, which are discussed in the framework of the above four conditions. It is preceded by some general results concerning uniformly continuous hull operators $(\S 2)$ to facilitate the discussion.

An important difference with the work of Michael, Curtis and Pasicki is the absence of real parameters describing convex combinations. Their proofs invariably use partitions of unity to get parameter values, leading to "approximate" selections or to "approximately continuous" selections. The impossibility to apply such methods in the current axiom system forced us to design a new approach. The idea is to use convex open sets to cut off pieces of the point-setvalues until each value set is reduced to a single point. How to cut is decided by a separate selection procedure in nerves of convex open covers. A key result is that such nerves are contractible. See $\S 4$.

This allows for techniques from homotopy theory in cell complexes, and it has several applications outside the framework of selections as well: fixed points (see 6.7, 6.8; see also [62] for a powerful Invariant Arc Theorem), and dimension theory (see 5.2; also: $[56,58]$ ). Applications of the selection theorem involve absolute retracts (5.1), Whitney levels (5.4), topological Hilbert cubes (5.5, 5.6, and also [59]), Beer's [2] approximation theorem for USC multifunctions (6.3), with an intermediate result on enlarging USC multifunctions to continuous multifunctions (6.1), and some results on lsc and usc functions in (semi)lattice theory $(6.5,6.6)$ which, perhaps, are most attractive to the reader who is knowledged in continuous (semi)lattice theory.

As the above list of results may illustrate, several applications have appeared elsewhere during the long period while this paper was submitted (the first manuscript is dated 1980). Several papers have been devoted to a deeper study of uniform convex structures (which are essentially the structures with property (iv) above). In [57], a study is made of uniform convex hyperspaces, the paper [62] gives a lot of information about spaces of arcs, and [52] discusses some problems on completion and compactification of uniform convex structures. Certain results of these papers have been used essentially here.

Several problems have been left open. One of the main results of [52] is that no totally bounded uniformity on $\mathbb{R}^{n}, n>1$ can make the standard hull operation uniformly continuous. Is it possible to find "exotic" uniformities in vector spaces of dimension $>1$, turning the standard hull operation uniformly continuous? 
A technical problem is whether the Kakutani Separation Property (see (iii) above) can be omitted from the list of assumptions of selection theory. This question is inspired by the observation that the condition is needed only in the preliminary result on contractibility of nerves of convex open covers, not in the actual construction of a selection.

A final type of problem is related with the fact that Michael and Curtis derive their selection theorem in circumstances where convex combinations need not exist for all finite sets. The equivalent viewpoint of synthetic convexity is what we call a convex system: a collection of subsets satisfying the axioms CONV 2 , 3 , with CONV 1 replaced by

CONV $1^{\prime}$. $\varnothing$ is convex (but not necessarily $X$ ).

The corresponding concept of a uniform convex system is developed in $\S 2$. For instance, spaces of arcs usually allow for a convex system only, but we have been able to show (3.10; also [62]) that these spaces extend to true convex structures in such a way that their convex sets remain convex in the extension (while preserving other relevant properties). A direct consequence is that the selection theorem applies to such convex systems (thus reproving Curtis' result). This leaves us with two more questions. First, is it possible to prove the selection theorem directly for (suitable) convex systems? Second, is it possible to extend other convex systems to convex structures in a way suggested above? This would lead to an indirect proof of a selection theorem for convex systems.

\section{Preliminaries}

1.1. Set-theoretic convexity. We refer to [22, 53 or 60$]$ for general information on convex structures. The axioms of convexity have been described in the introduction. We mostly omit explicit reference to the family of convex sets (the convexity). The convex hull of a set $A$ in a convex structure $X$ is defined by

$$
\operatorname{co}(A)=\bigcap\{C \mid A \subseteq C ; C \text { convex }\} .
$$

The hull of a finite set is a polytope. By the third axiom of convexity, a set is convex iff it includes the hull of each of its finite subsets.

A half-space of $X$ is a convex set with a convex complement. There is a list of separation axioms for convex structures, comparable with the conditions $T_{1}, \ldots, T_{4}$ in topology. Two of them are of current interest:

$\mathrm{S}_{1}$. All singletons are convex.

$\mathrm{S}_{4}$. Disjoint convex sets extend to complementary half-spaces.

The axiom $S_{1}$ is assumed valid throughout this paper.

A function $f: X \rightarrow Y$ between two convex structures is convexity preserving (CP) provided it inverts convex sets of $Y$ into convex sets of $X$.

1.2. Topological convexity. As far as topology is concerned, our notation and terminology is taken from $[19,14]$. The term map is used exclusively for continuous functions. Uniformities are described in terms of coverings.

A topological convex structure consists of a set, equipped with both a convexity and a topology, such that all polytopes are closed. If, in addition, the closure of each convex set is convex, then the structure is called closure stable. A concept of interior stability can be defined similarly. In [6, Theorem 2] it is shown that an ordinary compact convex set with its "relative" convexity need not be interior stable. This may illustrate that interior stability is not a frequent requirement, 
in contrast with closure stability (see the example list in $\S 3$, or see Theorem 2.4).

1.3. Convex systems. These are obtained by dropping the requirement that the entire space is a convex set. A subset $F$ of a convex system is called admissible provided it is included in some convex set of the system. Such sets constitute the domain of the hull operator of the system. As with convex structures, it is assumed that at least all singletons are convex.

Observe that a convex subset of a convex system is turned into a true convex structure relative to the family of its convex subsets. We will regard a convex system as a "system" of (mutually compatible) convex structures. Some terminology about convex structures can be adapted as follows. A convex system is $\mathrm{S}_{4}$ if each of its convex sets is $\mathrm{S}_{4}$. A topological convex system is one with an additional topology for which all polytopes (hulls of finite admissible sets) are closed.

1.4. Hyperspaces, multifunctions, and selections. Let $X$ be a topological space. Its hyperspace $H(X)$ is the collection of all nonempty closed subsets of $X$, equipped with the Vietoris (or finite) topology. If $A_{1}, \ldots, A_{n}$ are subsets of $X$, then $\left\langle A_{1}, \ldots, A_{n}\right\rangle$ denotes the collection

$$
\left\{C \in H(X) \mid C \subseteq \bigcup_{i=1}^{n} A_{i} ; \forall i=1, \ldots, n: C \cap A_{i} \neq \varnothing\right\} .
$$

A multifunction (or: a multivalued function) of $X$ to $Y$ is a relation $F \subseteq X \times Y$ such that the value sets

$$
F(x)=\{y \mid(x, y) \in F\} \quad(x \in X)
$$

are nonempty. We adopt the functional viewpoint, writing $F: X \multimap Y$.

For a set $O \subseteq Y$ we put

$$
F^{-1}(O)=\{x \mid F(x) \cap O \neq \varnothing\} ; \quad F^{\leftarrow}(O)=\{x \mid F(x) \subseteq O\} .
$$

If $X$ and $Y$ are spaces, then $F$ is lower semicontinuous (LSC) if $F^{-1}(O)$ is open in $X$ for each open set $O \subseteq Y . F$ is upper semicontinuous (USC) provided $F^{\leftarrow}(O)$ is open in $X$ for each open set $O \subseteq Y$. A multifunction which is both LSC and USC is called continuous for short. If all value sets of $F$ are closed in $Y$, then $F$ can be seen as a function $X \rightarrow H(Y)$, and the two resulting notions of continuity agree.

A selection of a multifunction $F: X \multimap Y$ is a function $f: X \rightarrow Y$ with $f(x) \in F(x)$ for all $x \in X$. If $A \subseteq X$ and $f: A \rightarrow Y$ satisfies $f(a) \in F(a)$ for all $a \in A$, then $f$ is called a partial selection of $F$ (over $A$ ).

\section{COMPATIBLE UNIFORM STRUCTURES}

2.1. Uniform convex structures. Let $(X, \mathscr{C})$ be a convex structure and let $\mu$ be a (covering) uniformity on $X$. We say that $\mu$ is compatible provided all polytopes are closed in the uniform topology and for each uniform cover $\mathscr{U} \in \mu$ there is a uniform cover $\mathscr{V} \in \mu$ such that

$$
\forall C \in \mathscr{C}: \operatorname{co}(\operatorname{st}(C, \mathscr{V})) \subseteq \operatorname{st}(C, \mathscr{U}) .
$$

The triple $(X, \mu, \mathscr{C})$ is called a uniform convex structure. The cover $\mathscr{V}$ with the property $(*)$ is referred to as the corresponding or the associated refinement of $\mathscr{U}$. 
A topological convex structure is uniformizable provided there is a uniformity generating the topology and which is compatible with the convexity.

We first give an equivalent description of compatibility. Recall $[19$, p. 572] that a uniformity $\mu$ on a space $X$ can be "lifted" to the hyperspace $H(X)$ in the following way. Let $\mathscr{U} \in \mu$. Two sets $A, B \in H(X)$ are $\mathscr{U}$-close provided $A \subseteq \operatorname{st}(B, \mathscr{U})$ and $B \subseteq \operatorname{st}(A, \mathscr{U})$. The sets of type

$$
[A, \mathscr{U}]=\{B \in H(X) \mid A, B \text { are } \mathscr{U} \text {-close }\}, \quad A \in H(X),
$$

form a covering of $H(X)$, and these covers constitute a uniformity base in $H(X)$. Note that on the subspace $H_{c}(X)$ of all compact sets, the uniform topology equals the usual Vietoris topology.

2.2. Lemma. Let $X$ be a convex structure and let $\mathscr{U}, \mathscr{V}$ be two covers of $X$. Then the following assertions are equivalent.

(1) $\operatorname{co}(\operatorname{st}(C, \mathscr{V})) \subseteq \operatorname{st}(C, \mathscr{U})$ for all convex sets $C \subseteq X$.

(2) If $F, G \subseteq X$ are finite and $\mathscr{V}$-close, then $\operatorname{co}(F), \operatorname{co}(G)$ are $\mathscr{U}$-close.

Proof. Assume (1) and let $F, G \subseteq X$ be $\mathscr{V}$-close. Then $G \subseteq \operatorname{st}(F, \mathscr{V})$ and consequently

$$
\operatorname{co}(G) \subseteq \operatorname{co}(\operatorname{st}(\operatorname{co}(F), \mathscr{V})) \subseteq \operatorname{st}(\operatorname{co}(F), \mathscr{U}) .
$$

Replacing the role of $F, G$ then yields (2).

Assume (2) and let $C \subseteq X$ be convex. If $x \in \operatorname{co}(\operatorname{st}(C, \mathscr{V}))$ then there is a finite set $F \subseteq \operatorname{st}(C, \mathscr{V})$ with $x \in \operatorname{co}(F)$. For each $a \in F$ we obtain a point $b \in C$ such that $\{a, b\} \subseteq V$ for some $V \in \mathscr{V}$. In this way we obtain a finite set $G \subseteq C$ which is $\mathscr{V}$-close to $F$. Consequently, $\operatorname{co}(G)$ is $\mathscr{U}$-close to $\operatorname{co}(F)$ and in particular there is a point $u \in \operatorname{co}(G)$ and a $U \in \mathscr{U}$ such that $\{u, x\} \subseteq U$. We find that $u \in C$ is $\mathscr{U}$-close to $x$, and hence that $x \in \operatorname{st}(C, \mathscr{U})$.

We let $H_{\mathrm{fin}}(X)$ denote the subspace of $H(X)$ consisting all of finite sets. The last result yields:

2.3. Theorem. Let $X$ be a topological convex structure and let $\mu$ be a uniformity on $X$ generating the topology of $X$. Then $\mu$ is compatible iff the operator

$$
\text { co: } H_{\text {fin }}(X) \rightarrow H(X)
$$

is uniformly continuous.

2.4. Theorem. A uniformizable convex structure is closure stable.

Proof. Let $\mu$ be a compatible uniformity. For each $\mathscr{U} \in \mu$ we fix a cover $U^{\prime} \in \mu$, associated to $\mathscr{U}$. If $C \subseteq X$ is convex, then

$$
\bar{C}=\bigcap\{\operatorname{st}(C, \mathscr{U}) \mid U \in \mu\}=\bigcap\{\operatorname{co}(\operatorname{st}(C, \mathscr{U})) \mid U \in \mu\},
$$

from which it follows that $\bar{C}$ is convex.

2.5. Theorem. The following are equivalent for a compact Hausdorff topological convex structure $X$.

(1) $X$ is a uniform convex structure.

(2) The convex closure operator, defined by

$$
\operatorname{co}^{*}(A)=\bigcap\{C \mid A \subseteq C, C \text { convex closed }\} \quad(A \in H(X)),
$$

is continuous on $H(X)$. 
Proof. The continuity of the operator co on $H_{\text {fin }}$ and the third axiom of convexity imply that the nonempty closed convex sets of $X$ form a closed subset of $H(X)$. By the definition of uniform convexity, each compact convex set has a neighborhood base (in $X$ ), consisting of convex sets. The first assertion implies that co* $^{*}$ is LSC, the second one implies that co* $^{*}$ is USC [22, III.7, 8].

The operator $\mathrm{co}^{*}$ is uniformly continuous by the compactness of $H(X)$ and its restriction to $H_{\text {fin }}(X)$ equals co by the fact that polytopes are closed.

In regard of Theorem 2.4, we have $\operatorname{co}^{*}(A)=\mathrm{Cl}(\operatorname{co}(A))$ for each subset $A$ of a uniform convex structure. There is an example [22, p. 60] of a compact topological convex structure $X$ (a subset of the separable Hilbert space $l_{2}$, actually), such that the hull operator is continuous on $H_{\text {fin }}(X)$, whereas the convex closure operator fails to be continuous on the whole of $H(X)$.

2.6. Theorem. Let $(X, \mu, \mathscr{C})$ be a uniform convex structure. Then for each $U \in \mu$ there is a $V \in \mu$ with the following property. If $C \subseteq X$ is convex, then there is a convex open set $O \subseteq X$ such that

$$
\operatorname{st}(C, \mathscr{V}) \subseteq O \subseteq \operatorname{st}(C, \mathscr{U}) .
$$

Proof. We fix two sequences of covers $\left(\mathscr{U}_{n}\right)_{n=0}^{\infty}$ and $\left(\mathscr{V}_{n}\right)_{n=0}^{\infty}$, where $\mathscr{U}_{0}=\mathscr{U}$ $\mathscr{U}_{n+1}$ is a star refinement of $\mathscr{U}_{n}$, and $\mathscr{V}_{n}$ is associated to $\mathscr{U}_{n+1}$ for each $n$. We verify that $\mathscr{V}=\mathscr{V}_{0}$ is as required. To this end, let $C=C_{0}$ be convex and inductively define $C_{n+1}=\operatorname{co}\left(\operatorname{st}\left(C_{n}, \mathscr{V}_{n}\right)\right)$. Then

$$
C_{n} \subseteq \operatorname{Int}\left(C_{n+1}\right) ; \quad C_{n+1} \subseteq \operatorname{st}\left(C_{n}, \mathscr{U}_{n+1}\right) .
$$

The second formula yields that

$$
\left.\operatorname{st}\left(C_{n+1}, \mathscr{U}_{n+1}\right) \subseteq \operatorname{st}\left(\operatorname{st}\left(C_{n}, \mathscr{U}_{n+1}\right), \mathscr{U}_{n+1}\right)\right) \subseteq \operatorname{st}\left(C_{n}, \mathscr{U}_{n}\right) .
$$

Therefore,

$$
\bigcup_{n=0}^{\infty} C_{n} \subseteq \bigcup_{n=0}^{\infty} \operatorname{st}\left(C_{n}, \mathscr{U}_{n}\right)=\operatorname{st}\left(C_{0}, \mathscr{U}_{0}\right) .
$$

The set on the left is convex open and is in between $\operatorname{st}\left(C_{0}, \mathscr{V}_{0}\right)$ and $\operatorname{st}\left(C_{0}, \mathscr{U}_{0}\right)$.

A topological convex structure is called (properly) locally convex if each of its points has a neighborhood base consisting of convex (open) sets. See [21 and 30], where a slightly different terminology is used for compact convex sets in vector spaces. The previous result implies that a uniformizable convex structure is properly locally convex. See 3.1 for a further discussion.

2.7. Theorem. Let $X$ be a uniform $\mathrm{S}_{4}$ convex structure. Then each two nonproximate convex sets can be separated by a uniformly continuous $C P$ function $f: X \rightarrow[0,1]$.

Proof. Let $\mu$ be the given uniformity on $X$, and let $C, D$ be convex sets which are nonproximate, that is:

$$
\exists \mathscr{U} \in \mu: \operatorname{st}(C, \mathscr{U}) \cap \operatorname{st}(D, \mathscr{U})=\varnothing .
$$

Let $\mathscr{V} \in \mu$ be associated to $\mathscr{U}$ as in 2.6. This gives us two convex open sets $O, P$ of $X$ with

$$
\operatorname{st}(C, \mathscr{V}) \subseteq O \subseteq \operatorname{st}(C, \mathscr{U}) ; \quad \operatorname{st}(D, \mathscr{V}) \subseteq P \subseteq \operatorname{st}(D, \mathscr{U}) .
$$


We find that $O \cap P=\varnothing$ and by the axiom $\mathrm{S}_{4}$ there is a half-space $H$ with the properties $O \subseteq H ; P \cap H=\varnothing$. For convenience, we label $H$ as $H_{1 / 2}$. Note that both pairs of convex sets $\left(C, X \backslash H_{1 / 2}\right) ;\left(H_{1 / 2}, D\right)$ are nonproximate (use a uniform star refinement of $\mathscr{V}$ ), and hence that the above "screening" process can be iterated. In a routine way, this leads to a collection of half-spaces $H_{t}$ for $0<t<1$ dyadic, such that the following hold for all dyadic numbers $s, t$.

(1) $H_{t}$ is increasing with $t$.

(2) Both pairs $\left(C, X \backslash H_{t}\right)$ and $\left(H_{t}, D\right)$ are nonproximate.

(3) If $s<t$ then the pair $\left(H_{s}, X \backslash H_{t}\right)$ is nonproximate.

Define a function $f: X \rightarrow[0,1]$ by the prescription

$$
f(x)=\inf \left\{t \mid x \in H_{t}\right\} .
$$

It is understood that in the right-hand expression, $t$ is a dyadic variable and the infimum of the empty set is 1 . In particular, $f$ maps $C$ to 0 and $D$ to 1 by (2). By (1), we find that

$$
f(x)=\sup \left\{t \mid x \notin H_{t}\right\},
$$

(with $\sup (\varnothing)=0$ ), and it is not difficult now to conclude that the sets $f^{-1}[0, r]$, $f^{-1}[r, 1]$ are convex for each $r \in[0,1]$. This shows $f$ to be CP. On the other hand, a uniform cover of $[0,1]$ admits a Lebesgue number $t=2^{-n}$. To see that $f$ is uniformly continuous, it suffices to verify that the sets $f^{-1}[k t,(k+2) t]$ for $k=0,1, \ldots, 2^{n}-2$ constitute a uniform cover of $X$. This is immediate from (2) and (3).

The nonproximity condition in 2.7 reflects the well-known criterion in vector spaces that two convex sets can be separated by a continuous linear functional iff the origin is not adherent to the algebraic difference of the sets.

2.8. Theorem. Let $X$ be a uniform convex structure with compact polytopes. Then the convex hull of a totally bounded set is totally bounded. If $X$ is uniformly complete, then the closed convex hull of a compact set is compact.

Proof. Let $A \subseteq X$ be totally bounded and let $\mathscr{U}$ be a uniform cover of $X$. Consider a sequence of uniform covers $\mathscr{V} \leq \mathscr{U}^{\prime} \leq \mathscr{U}$, where $\mathscr{U}^{\prime}$ is a star refinement of $\mathscr{U}$ and $\mathscr{V}$ is associated to $\mathscr{U}^{\prime}$. Fix a finite set $F \subseteq A$ with $A \subseteq \operatorname{st}(F, \mathscr{V})$. Then,

$$
\operatorname{co}(A) \subseteq \operatorname{co}(\operatorname{st}(F, \mathscr{V})) \subseteq \operatorname{st}\left(\operatorname{co}(F), \mathscr{U}^{\prime}\right) .
$$

Polytopes being compact, a finite subcover $U_{1}^{\prime}, \ldots, U_{n}^{\prime}$ of $\mathscr{U}^{\prime}$ can be found on $\operatorname{co}(F)$. After choosing $U_{i} \in \mathscr{U}$ such that $\operatorname{st}\left(U_{i}^{\prime}, \mathscr{U}^{\prime}\right) \subseteq U_{i}$ for $i=1, \ldots, n$, we find that

$$
\operatorname{co}\left(A j \subseteq \operatorname{st}\left(\operatorname{co}(F), \mathscr{U}^{\prime}\right) \subseteq \bigcup_{i=1}^{n} U_{i} .\right.
$$

This shows that $\operatorname{co}(A)$ is totally bounded; the second half of the theorem follows easily.

For results on completion of uniform convex structures, see [52].

2.9. Definitions. If $(X, \mathscr{C})$ is a convex system and if $\mu$ is a uniformity on $X$, then $\mu$ is compatible provided all polytopes are closed in the uniform topology, and for each $\mathscr{U} \in \mu$ there is a $\mathscr{V} \in \mu$ such that $\mathscr{V}$-close admissible sets have 
$\mathscr{U}$-close hulls. Compare with the formulation in Lemma 2.2. In these circumstances, the triple $(X, \mu, \mathscr{C})$ is called a uniform convex system. Note that each convex subspace of the system is turned into uniform convex structure, and that the various hull operators of such subspaces are equi-uniformly continuous. A topological convex system admitting a uniformity as above, inducing the given topology, is called uniformizable.

A uniform convex structure (system) $(X, \mathscr{C})$ is called metric provided the uniformity is generated by a metric $d$; then the topological convex structure (system) $(X, \mathscr{C})$ is called metrizable and the metric $d$ is said to be compatible.

For $A \subseteq X$ nonempty and $r>0$, we write $B(A, r)=\{x \mid d(x, A)<r\}$. By definition, $d$ is compatible iff for each $r>0$ there is an $s>0$ with co $B(C, s) \subseteq B(C, r)$ for each nonempty convex set $C$. According to Theorem 2.6, it can be taken care of that some convex open set is in between $B(C, s)$ and $B(C, r)$. In general, the open balls themselves need not be convex (not even the balls centered at one point).

\section{EXAMPLES}

3.1. Linear spaces. The traditional concept of a convex set in a linear space $X$ leads to the standard convexity on $X$, which is $\mathrm{S}_{4}$ by an old result of Kakutani [24]. A topology on $X$ making vector addition and scalar multiplication continuous turns this convexity into a topological one with compact polytopes and with connected convex sets.

If $X$ is locally convex, then the canonical translation-invariant uniformity is compatible with the standard convexity: let $\mathscr{U}$ be the cover of all translates of a neighborhood $U$ of the origin 0 . If $V, W$ are neighborhoods of 0 such that $V \subseteq U$ is convex and $W-W \subseteq V$, then $\operatorname{st}(C, \mathscr{W}) \subseteq C+V \subseteq \operatorname{st}(C, \mathscr{U})$, where $\mathscr{W}$ denotes the cover of all $W$-translates and the set $C+V$ is convex. Conversely, if some uniformity on $X$ is compatible with the standard convexity, then $X$ has a basis of convex open sets by Theorem 2.6 , whence $X$ is locally convex. Note that the compatible uniformity need not be the canonical one. For $X=\mathbb{R}$, the uniformity based on all finite convex open covers and the treeuniformities advocated in 3.8 below are compatible as well. It is not known whether there exist other compatible uniformities on locally convex linear spaces of higher dimension. A partial (negative) answer is given in [52]. Finally, a metrizable linear space admits a translation-invariant metric, [26, p. 48], and hence the standard convexity of a locally convex metrizable convex space is metrizable.

Let $X$ be a compact convex set in a topological vector space, equipped with the relative convexity. If $X$ is (intrinsically) locally convex, then [30 or 44] $X$ can be affinely embedded into a locally convex linear space. Consequently, such an $X$ is uniformizable as a convex structure. No direct proof of this fact is known yet. A corresponding result for noncompact convex subsets is not known to be valid.

3.2. Michael-Curtis and Pasicki convexity. A convex structure in the sense of Michael [35] consists of a metric space $(X, d)$, together with a sequence of functions

$$
k_{n}: M_{n} \times \Delta^{n-1} \rightarrow X \quad(n=1,2, \ldots),
$$


where $M_{n}$ is a subspace of $X^{n}$ including the diagonal and $\Delta^{m}$ is the standard $m$-simplex. The following conditions are assumed. For an $n$-tuple $u$ we use $\partial_{i}(u)$ to denote the $(n-1)$-tuple, obtained from $u$ by deleting the $i$ th entry.

(1) $k_{1}(x, 1)=x$

(2) If $x \in M_{n}$ then $\partial_{i}(x) \in M_{n-1}$, and if $t_{i}=0$ then $k_{n}(x, t)=$ $k_{n-1}\left(\partial_{i} x, \partial_{i} t\right)$.

(3) If $x \in M_{n}$ and $x_{i}=x_{i+1}$ then (with $t=\left(t_{1}, \ldots, t_{n}\right)$ ),

$$
k_{n}(x, t)=k_{n-1}\left(\partial_{i} x, t_{1}, \ldots, t_{i}+t_{i+1}, \ldots, t_{n}\right),
$$

(4) Each function $k_{n}$ is continuous in the variable $t \in \Delta^{n-1}$.

(5) If $\varepsilon>0$ then for each $n \in \mathbb{N}$ and $x \in M_{n}$ there is a $\delta=\delta(x)>0$ with $d\left(k_{n}(x, t), k_{n}\left(x^{\prime}, t\right)\right)<\varepsilon$ whenever $x^{\prime} \in M_{n}$ is $\delta$-close to $x$ and $t \in \Delta^{n-1}$ (equi-continuity in $x$ ).

Essentially, Curtis' definition [10] differs only in omitting condition (3) and in requiring equi-uniform continuity in (5). The functions $k_{n}$ are interpreted as "convex combinations" and following Michael or Curtis, a subset is convex if convex combinations of its points exist and are in the set. Note that this gives a convex system.

The main difficulty in comparing this notion of convexity with our lies in the fact that the initial emphasis is on certain external operations, rather than on the convex sets. In fact, one may wonder whether there have to exist nontrivial convex sets under the assumptions (1)-(5), even if $M_{n}=X^{n}$ for all $n$.

What can be said is that the convex hull operator of a Michael convexity need not be continuous. Take, for instance, a compact metric absolute retrace (AR) which is not locally an AR, [5]. As with any AR, $X$ admits a Michael convexity, obtained from embedding $X$ in a Banach space as a closed subset of its convex hull, [19], and by "retracting" convex combinations back to $X$. Now, let $p \in X$ be a point where $X$ is not locally an AR. Specifically, there is a neighborhood $U$ of $p$, no subneighborhood of which is an AR. If the convex hull operator $H_{\mathrm{fin}}(X) \rightarrow H(X)$ is continuous at $\{p\}$, then there is a neighborhood $V$ of $p$ with $\operatorname{co}(F) \subseteq U$ for each finite set $F \subseteq V$, meaning that $\operatorname{co}(V) \subseteq U$. One can even take care that $\mathrm{Cl}(\operatorname{co}(V)) \subseteq U$. However, any Michael convexity is closure stable (by virtue of $(5)$ ), and the convex set $\mathrm{Cl}(\operatorname{co}(V))$ is an $\mathrm{AR},[35, \mathrm{p}$. 559], a contradiction.

Both Michael and Curtis use convexity on the exclusive purpose of obtaining a selection theorem. A different notion of "parameterized" convexity has recently been proposed by Pasicki, [41]. Essentially, it consists of one function of type $k_{2}$ only, defined on the whole of $X^{2}$. Pasicki obtains a selection theorem under the additional assumption that a certain hull-like operator is uniformly continuous. As with the Michael-Curtis convexity, the explicit introduction of parameterized convex combinations makes it difficult to compare this notion with ours.

3.3. Median convexity. In lattice theory one frequently encounters the convexity consisting of all order-convex sublattices. It was shown in $[55,3.5]$ that this convexity is $\mathrm{S}_{4}$ iff the lattice is distributive. A basic ingredient in proving this is the classical theorem of Stone on separation of lattice points by prime ideals. See [18, p. 418] for a unified proof of Stone's and Kakutani's (see 3.1) separation theorems. 
The Helly number of a convex structure is defined to be the least $n \in \mathbb{N}$ such that every finite family of convex sets meeting $n$ by $n$ has a nonempty intersection. The above described convexity on a lattice has a Helly number $\leq 2$. The median operator of a distributive lattice $(L, \wedge, \vee)$ is the function

$$
m: L^{3} \rightarrow L ; \quad m(x, y, z)=(x \wedge y) \vee(y \wedge z) \vee(z \wedge x) .
$$

A subset $X \subseteq L$ is called median-stable provided $m\left(X^{3}\right) \subseteq X$. Somewhat surprisingly, the class of all $\mathrm{S}_{4}$ convex structures of Helly number $\leq 2$ can be characterized in first-order terms as the class of median-stable subsets of distributive lattices (with relative convexity), $[55,3.6]$.

Compact trees, where connected sets are taken convex, are perhaps the simplest type of example (see 3.8 for a discussion of not necessarily compact trees). The superextension of a $\mathrm{T}_{4}$ space $X$ is the space $\lambda(X)$, whose points are the maximal families of pairwise intersecting closed sets of $X$, with a Wallmantype topology given as follows. If $A \subseteq X$ is closed, then $A^{+}$is the set of all $l \in \lambda(X)$ with $A \in l$. The sets of this type are taken as a closed subbase. The resulting space is compact Hausdorff, and the above described subbasic sets can also be used to generate a convexity on $\lambda(X)$, which is of the median type.

Two more classes of examples arise from the following recent results. Among the Banach spaces with "metric convexity" the $L_{1}(\mu)$-spaces are precisely the median ones, [64, 4.6]. In [61], the author characterized collapsible cubical polyhedra in terms of the existence of a median convexity on the underlying polyhedron.

A locally convex, median, $\mathrm{S}_{4}$ convexity on a compact space $X$ has two more properties of interest:

(1) If $X$ is connected, then so is each convex subset of $X,[38,2.5]$.

(2) If $X$ is (topologically) metrizable, then there is a metric on $X$ with $B(C, r)$ convex for each convex set $X$ and $r>0,[37,3.6]$. Hence $X$ is metrizable as a convex structure.

3.4. Semilattices. Let $(S, \wedge)$ be a (meet) semilattice. The collection of all order-convex subsemilattices is a convexity on $S$ which will be considered throughout. See [23] for other possible convexities on a semilattice. The canonical convexity has the Kakutani separation property.

This can be seen as follows. For $A \subseteq S$ we denote

$$
\leq(A)=\{x \mid x \leq a\} ; \quad \geq(A)=\{x \mid x \geq a\} .
$$

Let $C, D \subseteq S$ be disjoint convex subsets of $S$ and suppose that $\leq(C \cap D)$ and $C \cap \leq(D)$ are both nonempty. This gives us four points $c_{1}, c_{2} \in C, d_{1}, d_{2} \in D$ with $d_{1} \leq c_{1}$ and $c_{2} \leq d_{2}$. As $C, D$ are semilattices, we find that the point $c=c_{1} \wedge c_{2}$ is in $C$ and that the point $d=d_{1} \wedge d_{2}$ is in $D$. As $C, D$ are order-convex, we conclude from the inequalities

$$
d \leq c_{1} \wedge d_{2} \leq d_{2} ; \quad c \leq c_{1} \wedge d_{2} \leq c_{1}
$$

that $c_{1} \wedge d_{2}$ is common to $C, D$, contradictory to the assumption. So assume $\leq(C) \cap D=\varnothing$. Note that then $C \cap \geq(D)=\varnothing$. The set $\geq(D)$ is easily seen to be a half-space; it separates between $C$ and $D$.

Let $S$ now be a compact $\mathrm{T}_{2}$ semilattice. In particular, the meet operator $S \times S \rightarrow S$ is assumed continuous. Then $S$ clearly is a topological convex 


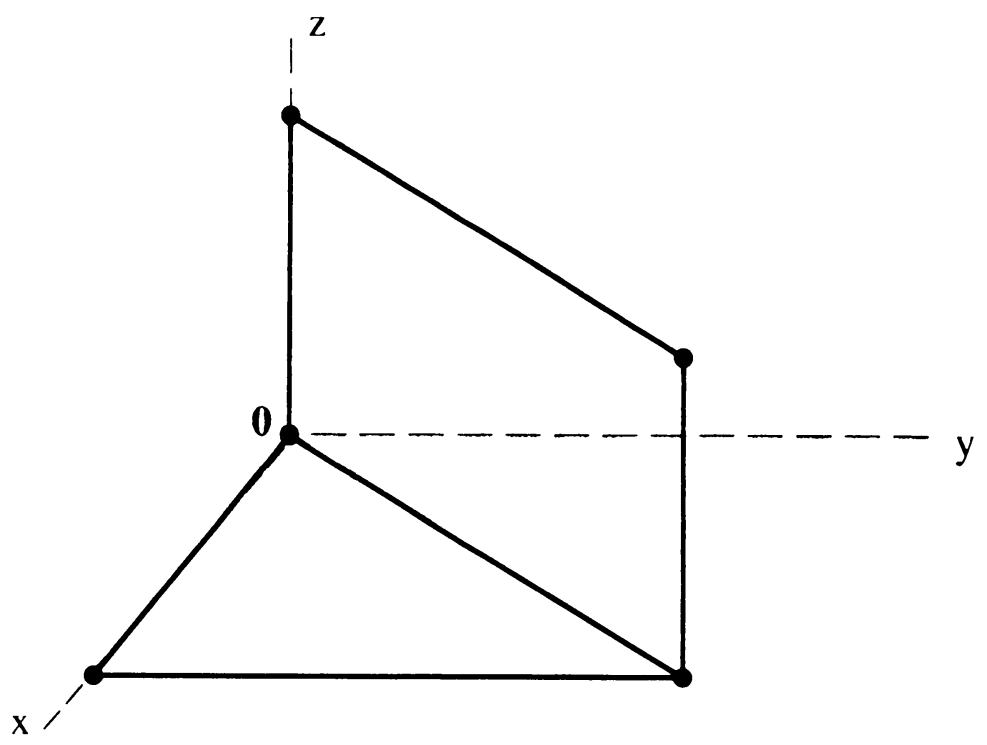

FIGURE 1. A semilattice with a discontinuous interval operator

structure. The following conditions on $S$ are equivalent [20, pp. 273, 284285]:

(1) $S$ has small lattices (or: is a Lawson semilattice), that is: each point has a neighborhood base consisting of subsemilattices.

(2) $S$ is locally convex.

(3) The operator $H(S) \rightarrow S ; A \mapsto \inf (A)$, is continuous.

The convex closure of a compact set $A \subseteq S$ equals $\{x \mid \inf (A) \leq x \leq a$ for some $a \in A\}$. This may suggest that the convex closure operator is continuous iff $S$ is locally convex. However, the set

$$
S=\{(x, y, z) \mid z=0,0 \leq y \leq x \leq 1, \text { or, } 0 \leq x=y \leq 1,0 \leq z \leq 1\}
$$

describes a compact subsemilattice of the 3-cube (coordinate-wise meet). See Figure 1. The convex closure operator of this locally convex space fails to be continuous at subsets of the edge joining the origin with the point $(1,1,0)$.

Apparently, the behavior of the intervals $[a, b]=\{x \mid a \leq x \leq b\}$ for $a \leq b$ in $S$ has to be incalculated: $S$ has a continuous convex closure operator iff it is locally convex and the intervals $[a, b]$ depend continuously on the pair of endpoints $a \leq b$.

As a final point of interest, all convex sets in a compact semilattice $S$ are connected iff $S$ is arcwise connected (an arc being a totally ordered subcontinuum; see also 3.10).

3.5. Lattices. We have already touched upon a convexification of lattices in 3.3. The resulting structure is particularly useful in case the lattice is completely distributive. In compact $T_{2}$ lattices which fail to have that property, a second choice is to consider the convexity of all order-convex meet subsemilattices.

In this convexity, intervals do depend continuously on their endpoints and hence-as observed in 3.4-the proposed convexity is uniform iff the lattice has small meet semilattices. Moreover, the convexity is $\mathrm{S}_{4}$ (cf. 3.4), regardless of 
whether the distributive property holds. Finally, all convex sets are connected if the original lattice is [23, p. 365].

3.6. Convex hyperspaces: the semilattice viewpoint. If $(X, \mathscr{C})$ is a topological convex structure, then $C(X, \mathscr{C})$ denotes the subspace of $H(X)$, consisting of all compact convex sets in $X$. The resulting space is known as the convex hyperspace of $(X, \mathscr{C})$, [57]. The space $C(X, \mathscr{C})$ can be seen as a semilattice in circumstances where the closed convex hull of the union of two compacta $C, D$ is compact: the "meet" is $\operatorname{co}^{*}(C \cup D)$. This is the case e.g. if $(X, \mathscr{C})$ is completely uniformizable (Theorem 2.8 ). The simplest situation occurs when $X$ is merely compact and the convex closure operator is continuous on the hyperspace of all compacta (Theorem 2.5). Then $C(X, \mathscr{C})$ is compact (being a retract of $H(X))$. In addition, it has small semilattices (see also [48]), and its intervals depend continuously on their endpoints. Hence, by the discussion in 3.4, the semilattice $C(X, \mathscr{C})$ is a uniform convex structure. By [23, p. 369], it is arcwise connected iff all convex sets in $X$ are connected.

3.7. Convex hyperspaces: the Vietoris point of view. For a (general) topological convex structure $(X, \mathscr{C})$ there is a different way to convexify $C(X, \mathscr{C})$. With the notation of 1.4 , the collection of all sets of type

$$
\langle C\rangle \cap C(X, \mathscr{C}) \text { or }\langle C, X\rangle \cap C(X, \mathscr{C})
$$

(where $C \subseteq X$ is convex closed) generates a convexity on $C(X, \mathscr{C})$ which is said to be induced by $\mathscr{C},[57,1.1]$. According to [57], a compact convex set $A$ is in the convex hull of $A_{1}, \ldots, A_{n} \in C(X, \mathscr{C})$ iff $A$ is included in the convex closure of $\bigcup_{i=1}^{n} A_{i}$ and $A$ meets $\operatorname{co}\left\{a_{1}, \ldots, a_{n}\right\}$ for each choice of $a_{i} \in A_{i}$. A compatible uniformity on $X$ can be lifted to a compatible uniformity on $C(X, \mathscr{C})$ provided the convex closure of the union of two compacta is compact. In particular, metrizability of convex structures is preserved when passing to convex hyperspaces. Other preserved properties are: $S_{4}$, compactness of polytopes, and connectedness of convex sets. See [57, 1.3, 1.5].

The induced convexity has properties quite distinct from those of the semilattice convexity. For instance, its Helly number (cf. 3.3) is usually much lower, and it inherits certain properties from the base space which a semilattice convexity almost never possess; see [54] for details. The main advantage of the present construction over the one in 3.6 is, that compactness of the base space is not required in certain applications (compare 6.1 and 6.5 below). On the other hand, a semilattice convexity is always $\mathrm{S}_{4}$ (see 3.4 ) whereas for the induced convexity, this depends on whether or not the original convexity is $S_{4}$.

3.8. Topological trees. Recall that a tree-like space is a connected Hausdorff space in which every two distinct points can be separated with a third one. A tree is a locally connected tree-like space. A compact tree-like space is automatically locally connected, [65, Lemma 4]. The collection of all connected subsets of a tree turns out to be a median convexity, [53, 2.10].

We now show that if a tree $X$ is completely regular, then the corresponding convex structure is uniformizable. Let $\mu$ be any uniformity on $X$ (there is one by complete regularity). For $\mathscr{U} \in \mu$, let $\mathscr{U}^{\prime}$ denote the open cover, consisting of all components of members of $\mathscr{U}$. The coverings so obtained constitute a basis for another uniformity $\mu^{\prime}$, which is easily seen to be compatible with the tree convexity. Note that $\mu^{\prime}$ is complete if $\mu$ is. If $d$ is a metric for $\mu$, then 
$\mu$ has a countable base of coverings $\mathscr{U}$, and the corresponding coverings $\mathscr{U}^{\prime}$ then yield a countable base for $\mu^{\prime}$. It follows that $\mu^{\prime}$ is metric too, [19, p. 533].

3.9. Uniform quotients of convex structures. The procedure which we describe next has been introduced in [39] to obtain results in dimension theory. Let $(X, \mu, \mathscr{C})$ be a uniform convex structure, and let $\mu^{\prime}$ be a subuniformity of $\mu$. Suppose that each cover in $\mu^{\prime}$ has an associated refinement (in the sense of Definition 2.1) in $\mu^{\prime}$. Let ' $\equiv$ ' denote the equivalence relation on $X$ determined by $\mu^{\prime}$, and let $q: X \rightarrow \widetilde{X}=X / \equiv$ be the quotient map. Put

$$
\tilde{\mathscr{C}}=\left\{C \mid q^{-1}(C) \in \mathscr{C}\right\} ; \quad \tilde{\mu}=\left\{U \mid q^{-1}(U) \in \mu^{\prime}\right\} .
$$

It was shown in $[39, \S 2]$ that

(1) $(\tilde{X}, \tilde{\mu}, \tilde{\mathscr{C}})$ is a uniform convex structure.

(2) $\widetilde{\mathscr{C}}$ is $\mathrm{S}_{4}$ provided $\mathscr{C}$ is.

(3) $\tilde{\mathscr{C}}$ has compact polytopes provided $\mathscr{C}$ has.

(4) The direct and inverse image under $q$ of a convex set are convex.

For compact $X$, such subuniformities are completely determined by a USC equivalence relation on $X$ with closed equivalence classes, such that the saturation of a convex set is convex.

Figure 2 illustrates how a tree (the "character $Y$ ") can be obtained as a quotient of the two-cell with its standard convexity. In fact, by complicating the method, any compact metric tree can be obtained as a quotient of the twocell.

A more effective way of constructing uniform quotients in convexity is provided by the "Factorization Theorem," [39, 3.1]. This result can be seen at work in 6.8 below.
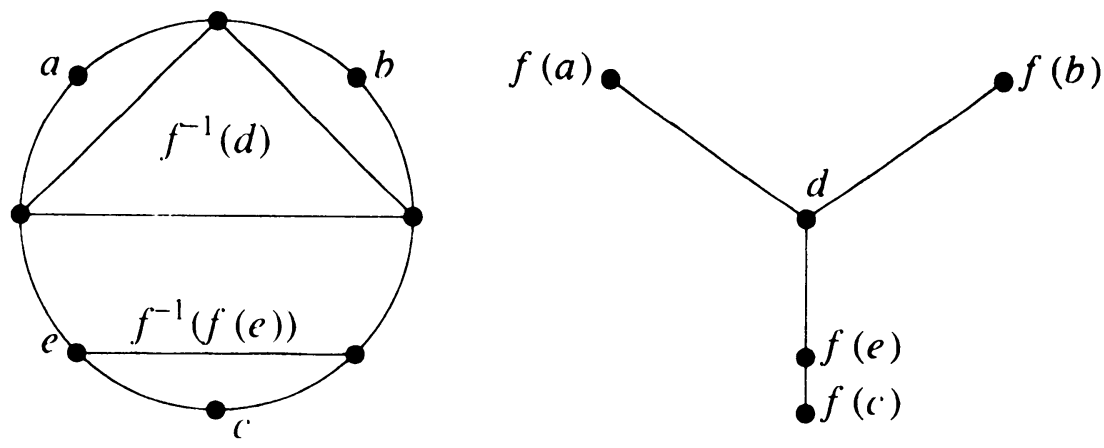

FIGURE 2. Uniform quotient of the two-cell

3.10. Spaces of arcs. An (order) arc in a partially ordered space $Y$ is a totally ordered subcontinuum. The set $\Gamma(Y)$ of all order arcs is topologized as a (closed) subspace of the hyperspace of compacta, $H_{c}(Y)$. Such spaces were considered by Eberhart, Nadler and Nowell in [16] for $Y=C(X)$, the hyperspace of subcontinua of a metric continuum $X$. We let $\Lambda(Y)$ denote the subspace of $\Gamma(Y)$, consisting of all maximal arcs. For $Y=C(X)$, this space was considered in [10].

A convex system can be constructed on $\Gamma(Y)$ as follows. Assume that each lower set $\leq(y)$ for $y \in Y$ is a topological meet sublattice. If $\alpha_{1}, \ldots, \alpha_{n} \in$ 
$\Gamma(Y)$ join the same pair of points in $Y$, then the hull of $\alpha_{1}, \ldots, \alpha_{n}$ is defined to be the set of all arcs $\alpha$ which are in between $\alpha_{1}, \ldots, \alpha_{n}$, that is: $\alpha$ joins the same pair of points and is built up with points of type

$$
a_{1} \wedge \cdots \wedge a_{n} \text {, with } a_{1} \in \alpha_{1}, \ldots, a_{n} \in \alpha_{n} .
$$

Taking into account the third axiom of convexity, one can verify that this prescription determines a convex system on $\Gamma(Y)$. Its convex sets live on orderconvex subspaces of $Y$ with a top and a bottom element.

In [10], a Michael-type convex system (see 3.2) was built on $\Lambda(C(X))$ for $X$ a metric continuum. Note that maximal arcs join some singleton to $X$. A "convex combination" can be taken only of a finite set of arcs, starting at the same singleton. The resulting arc is built up with points as in (1) above (the "meet operation" is simply the union of subcontinua).

We showed in [62] that if $S$ is an arcwise connected Lawson semilattice (see 3.4), then the resulting convex structure on $\Lambda(S)$ is uniform, $S_{4}$ (this is tricky!), that it has connected convex sets, and that its polytopes are compact. Moreover, the "modulus" of uniform continuity equals the one of the infimum operator $H(X) \rightarrow S$. For $Y=C(X)$, with $X$ a continuum, we have a family of arcwise connected Lawson semilattices, one for each pair of subcontinua $A \subseteq B$, with an equi-continuous set of "union" operators. It follows that our convex system on the Eberhart-Nadler-Nowell space is uniformizable (and even metrizable if $X$ is).

The following result states that a convex system of arcs can be extended to a convex structure in a way that makes the forthcoming selection theorem applicable. The term "subspace" refers to both topology and convexity.

Theorem $[62,3.5]$. Let $X$ be a continuum. Then there is a uniform $\mathrm{S}_{4}$ convex structure $Z$ with compact polytopes and with connected convex sets, together with a topological embedding of $\Gamma C(X)$ into $Z$ such that each convex subspace of $\Gamma C(X)$ is a convex subspace of $Z$. Moreover, $Z$ is metrizable if $X$ is.

\section{THE SELECTION THEOREM}

We briefly recall some conventions, notation, and terminology. See [46] for details. The simplices of a simplicial convex $S$ will be denoted by Greek $\sigma, \rho, \ldots$ and its vertex set is again denoted by $S$. We let $|S|$ be the geometric realization of $S$ and $|S|^{q}$ its $q$-skeleton. A simplicial function $\alpha: S \rightarrow T$ has a unique PL-extension $|\alpha|:|S| \rightarrow|T|$.

A family $\mathscr{O}$ of subsets of a set $X$ induces a simplicial convex with vertex set $\mathscr{O}$; its simplices are the finite subsets of $\mathscr{O}$ with a nonempty intersection. The geometric realization of $\mathscr{O}$ is called the nerve of $\mathscr{O}$. A simplex $\left\{O_{1}, \ldots, O_{n}\right\}$ of $\mathscr{O}$ is on a subset $A$ of $X$ provided $\bigcap_{i=1}^{n} O_{i} \cap A \neq \varnothing$. The simplices on $A$ constitute a subcomplex $\mathscr{O}(A)$ of $\mathscr{O}$.

If $\mathscr{P}$ is a second family of subsets of $X$, then a refinement function $\alpha$ : $\mathscr{P} \rightarrow \mathscr{O}$ is a simplicial function satisfying $P \subseteq \alpha(P)$ for each $P \in \mathscr{P}$. In these circumstances, $\mathscr{P}$ is called a refinement of $\mathscr{O}$, in symbols: $\mathscr{P} \leq \mathscr{O}$.

4.1. Theorem. Let $X$ be a closure stable and $\mathrm{S}_{4}$ convex structure with connected convex sets and with compact polytopes, let $C \subseteq X$ be nonempty convex, and let $\mathcal{O}$ be a family of convex open sets of $X$ covering $C$. Then the polyhedron $|\mathscr{O}(C)|$ is contractible. 
Proof. We proceed in two stages.

(I) The theorem holds for finite convex open covers of a convex set.

Let $\mathscr{O}$ be a finite collection of convex open (in $X$ ) sets covering $C$, and let $s$ be the total number of simplices occurring in $\mathscr{O}(C)$. If $s=1$ we are done. We proceed by induction as follows. Each simplex of $\mathscr{O}(C)$ extends to a maximal one. If only one maximal simplex occurs, then the result is evident. Suppose $\sigma_{1}$ and $\sigma_{2}$ are distinct maximal simplices. Then $\bigcap \sigma_{1} \cap C$ and $\bigcap \sigma_{2} \cap C$ are disjoint convex sets whence by the Kakutani separation property there is a half-space $H \subseteq X$ with

$$
\bigcap \sigma_{1} \cap C \subseteq H ; \quad \bigcap \sigma_{2} \cap C \subseteq X \backslash H .
$$

Let $C_{1}=\mathrm{Cl}(C \cap H) ; C_{2}=\mathrm{Cl}(C \backslash H)$. Then $\left|\mathscr{O}\left(C_{1}\right)\right| \cup\left|\mathscr{O}\left(C_{2}\right)\right|=\mathscr{O}_{C}$. On the other hand, if a simplex $\sigma$ is in $\mathscr{O}\left(C_{i}\right)$ for $i=1,2$ then the nonempty connected set $\cap \sigma$ meets $C_{1}, C_{2}$ and hence it meets $C_{1} \cap C_{2}$. This shows that $\mathscr{O}\left(C_{1}\right) \cap \mathscr{O}\left(C_{2}\right)=\mathscr{O}\left(C_{1} \cap C_{2}\right)$. Note that $\bigcap \sigma_{1}$ is disjoint with $C_{2}$ and that $\bigcap \sigma_{2}$ is disjoint with $C_{1}$ since $\bigcap \sigma_{1}, \bigcap \sigma_{2}$ are open sets. Consequently, the polyhedra $\mathscr{O}\left(C_{1}\right), \mathscr{O}\left(C_{2}\right), \mathscr{O}\left(C_{1} \cap C_{2}\right)$ each have less than $s$ simplices and by inductive assumption, they are contractible. Then $[5$, p. 90$]$ the polyhedron $\mathscr{O}(C)=\mathscr{O}\left(C_{1}\right) \cup \mathscr{O}\left(C_{2}\right)$ is contractible.

(II) The theorem holds for arbitrary convex open covers of a convex set.

Let $\mathscr{O}$ be a convex open covering of the convex set $C$, and let $K \subseteq|\mathscr{O}(C)|$ be compact. Then [46, p. 113] there is a compact polyhedron $P \subseteq|\mathscr{O}(C)|$ including $K$. Let $\mathscr{O}^{\prime}$ be the set of vertices of $P$. For each simplex $\sigma$ of $P$ we fix a point in $\bigcap \sigma \cap C$. The convex hull of the selected points is a compact subset $C_{0}$ of $C$ and it is covered by a finite subcollection $\mathscr{O}^{\prime \prime}$ of $\mathscr{O}$. For $\mathscr{P}=\mathscr{O}^{\prime} \cup \mathscr{O}^{\prime \prime}$, we find that $K \subseteq P \subseteq \mathscr{P}\left(C_{0}\right)$, and the last polyhedron is contractible by the first part of the proof. This shows that $|\mathscr{O}(C)|$ is weakly homotopy trivial and hence [46, p. 405] that this polyhedron is contractible.

We now proceed with a proof of a selection theorem. We first concentrate on multifunctions with a finite dimensional domain.

4.2. Proposition. Let $X$ be an $\mathrm{S}_{4}$ topological convex structure with compact polytopes and with connected convex sets, and let $d$ be a compatible metric. If $Y$ is a finite dimensional paracompact space and if $F: Y \rightarrow X$ is an LSC multivalued function with convex and $d$-complete value sets, then $F$ admits a continuous selection.

Proof. By Theorem 2.6, $X$ has a base of uniform convex open covers. Let $\mathscr{O}_{0}$ be such a cover and consider the multifunction

$$
S_{0}: Y \multimap\left|\mathscr{O}_{0}\right| ; \quad S_{0}(y)=\left|\mathscr{O}_{0}(F(y))\right| .
$$

The greater part of our efforts are spent on a proof of the following result.

Lemma. There is a continuous selection $s$ of $S_{0}$ with the following properties.

(i) If $q$ denotes $\operatorname{dim}(Y)$, then $s(Y) \subseteq\left|\mathscr{O}_{0}\right|^{q}$.

(ii) Each $y \in Y$ has a neighborhood $\bar{W}_{y}$ such that $s\left(W_{y}\right)$ is relatively compact.

Let $\mathscr{O}_{0}^{\prime}$ and $\mathscr{O}_{1}$ be uniform convex open covers of which the first one star refines $\mathscr{O}_{0}$ and the second one is associated to $\mathscr{O}_{0}^{\prime}$ as in $(*)$ of 2.1 . We consider 
a composed refinement map $\alpha_{1}: \mathscr{O}_{1} \stackrel{\gamma}{\rightarrow} \mathscr{O}_{0}^{\prime} \stackrel{\beta}{\rightarrow} \mathscr{O}_{0}$, where $\beta$ is chosen such that $\operatorname{st}\left(O^{\prime}, \mathscr{O}_{0}^{\prime}\right) \subseteq \beta\left(O^{\prime}\right)$ for all $O^{\prime} \in \mathscr{O}_{0}^{\prime}$. The multifunction $S_{1}: Y \rightarrow\left|\mathscr{O}_{1}\right|$ is defined by $S_{1}(y)=\left|\mathscr{O}_{1}(F(y))\right|$. Note that the refinement map $\left|\alpha_{1}\right|$ maps $S_{1}(y)$ into $S_{0}(y)$. We first verify the following.

(1) If $A \subseteq Y$ is nonempty and $\bigcap_{a \in A} S_{1}(a) \neq \varnothing$, then the restriction

$$
\bigcap_{a \in A} S_{1}(a) \rightarrow \bigcap_{a \in A} S_{0}(a)
$$

of $\left|\alpha_{1}\right|$ is null homotopic.

Indeed, if $a \in A$ then for each simplex $\sigma$ of $\bigcap_{a \in A} S_{1}(a)$ we can fix a point in $\bigcap \sigma \cap F(a)$, and we let $C(a)$ denote the hull of these points. We also fix a point $p \in A$ for reference. Now $C(a), C(p)$ are the hull of $\mathscr{O}_{1}$-close sets and hence are $\mathscr{O}_{0}^{\prime}$-close. Also, $\gamma$ maps $\bigcap_{a \in A} S_{1}(a)$ into $\mathscr{O}_{0}^{\prime}(C(p))$, which is contractible by Theorem 4.1.

So it suffices to show that $\beta$ maps the last named complex into $\bigcap_{a \in A} S_{0}(a)$. To this end, let $\sigma \subseteq \mathscr{O}_{0}^{\prime}$ be a simplex on $C(p)$ and fix a point $x \in \cap \sigma \cap C(p)$. For each $a \in A$ the set $C(a)$ is $\mathscr{O}_{0}^{\prime}$-close to $C(p)$ and hence there is a point $x_{a} \in C(a)$ such that $x, x_{a}$ occur together in some $O_{a}^{\prime} \in \mathscr{O}_{0}^{\prime}$. Hence for every $O^{\prime} \in \sigma$ we have $\beta\left(O^{\prime}\right) \supseteq \operatorname{st}\left(O^{\prime}, \mathscr{O}_{0}^{\prime}\right) \supseteq O^{\prime} \cup O_{a}^{\prime}$, showing that $x_{a} \in \bigcap \beta(\sigma)$. Hence the image simplex is in $\bigcap_{a \in A} S_{0}(a)$.

By repeating the above constructions, we obtain a sequence of uniform convex open covers and of refinement functions

$$
\mathscr{O}_{q} \stackrel{\alpha_{q}}{\longrightarrow} \mathscr{O}_{q-1} \longrightarrow \cdots \stackrel{\alpha_{1}}{\longrightarrow} \mathscr{O}_{0}
$$

where $q=\operatorname{dim} Y$, together with a sequence of multifunctions

$$
S_{j}: Y \multimap\left|\mathscr{O}_{j}\right| ; \quad S_{j}(y)=\left|\mathscr{O}_{j}(F(y))\right|, \quad j=0, \ldots, q,
$$

with the following property:

(2) If $A \subseteq Y$ is nonempty and if $\bigcap_{a \in A} S_{j+1}(a) \neq \varnothing$ then the restriction

$$
\bigcap_{a \in A} S_{j+1}(a) \rightarrow \bigcap_{a \in A} S_{j}(a)
$$

of $\left|\alpha_{j+1}\right|$ is homotopy trivial.

As $F$ is LSC, we obtain an open cover

$$
F^{-1}(\mathscr{O})=\left\{F^{-1}(O) \mid O \in \mathscr{O}\right\}
$$

of $Y$, which admits a $q$-dimensional open refinement $\mathscr{V}$. We now construct a commutative diagram of maps as indicated below.

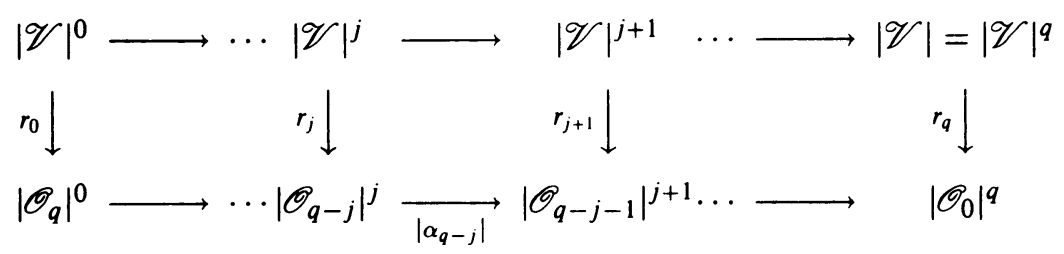

For each simplex $\sigma \subseteq \mathscr{V}$, define a subset of $Y$ by $A(\sigma)=\bigcap \sigma$. Note that $A(\sigma) \neq \varnothing$. For each $V \in \mathscr{V}$, choose $O_{V}$ in such a way that $V \subseteq F^{-1}\left(O_{V}\right)$, and put $r_{0}(V)=O_{V}$. Evidently, 
(3;0) For each simplex $\sigma \subseteq \mathscr{V}$, the function $r_{0}$ maps $|\sigma|^{0}$ into the 0 skeleton of $\bigcap_{a \in A(\sigma)} S_{q}(a)$.

We proceed by induction to construct mappings $r_{j}$ for $j \leq q$ as suggested in the diagram and such that

$(3 ; j)$ For each simplex $\sigma \subseteq \mathscr{V}$, the function $r_{j}$ maps $|\sigma|^{j}$ into the $j$ skeleton of $\bigcap_{a \in A(\sigma)} S_{q-j}(a)$. For $j<q$, the step $j \mapsto j+1$ is made as follows. Let $\tau \subseteq \mathscr{V}$ be a $(j+1)$-simplex. Then the composition of its restriction $|\tau|^{j} \rightarrow\left(\bigcap_{a \in A(\tau)} S_{q-j}(a)\right)^{j}$ with $\alpha_{q-j}$ is null homotopic in the complex $\bigcap_{a \in A(\tau)} S_{q-j-1}(a)$, and hence within its $(j+1)$-skeleton (cellular approximation theorem, [46, p. 404]). This homotopy determines $r_{j+1}$ on $\tau$, and fitting these pieces of maps together yields a mapping $r_{j+1}$ as suggested. Statement $(3 ; j+1)$ is clear for simplices $\sigma$ of dimension $\leq j+1$. Suppose $\sigma$ is of dimension $>j+1$. If $\tau$ is a $(j+1)$-face of $\sigma$ we have $A(\tau) \supseteq A(\sigma)$, whence

$$
\bigcap_{a \in A(\tau)} S_{q-j-1}(a) \subseteq \bigcap_{a \in A(\sigma)} S_{q-j-1}(a) .
$$

Hence $|\sigma|^{j+1}$ is mapped into the $(j+1)$-skeleton of the last complex.

This completes the construction of the diagram, producing a mapping $r=r_{q}$. By the paracompactness of $Y$, there is a partition of unity $\left(g_{V}\right)_{V \in \mathscr{V}}$ subordinate to $\mathscr{V},\left[19\right.$, p. 374]. For $y \in Y$ we fix a neighborhood $W_{y}$ on which only finitely many $g_{V}$ do not vanish. We obtain a mapping $g: Y \rightarrow|\mathscr{V}|$ with the following property.

(4) For each $y \in Y$, the set $g\left(W_{y}\right)$ is included in a compact full subcomplex of $|\mathscr{V}|$ built with the vertices $V$ satisfying $\operatorname{supp}\left(g_{V}\right) \cap W_{y} \neq \varnothing$.

The desired selection of $S_{0}$ is defined as the composition $r \circ g$. We check its properties. Let $y \in Y$ and let $\sigma=\left\{V_{0}, \ldots, V_{p}\right\} \quad(p \leq q)$ be the smallest simplex of $V$ with $g(y) \in|\sigma|$. Then $y_{i} \in \bigcap_{i=0}^{p} V_{i}=A(\sigma)$. By $(3, q)$, we find that $\operatorname{rg}(y) \in\left(\bigcap_{a \in A(\sigma)} S_{0}(A)\right)^{q} \subseteq S_{0}(y)^{q}$, showing that $s=\mathrm{rg}$ is a selection of $S_{0}$ ranging into the $q$-skeleton of $\left|\mathscr{O}_{0}\right|$. By (4), we also conclude that $s\left(W_{y}\right)$ is relatively compact in $\left|\mathscr{O}_{0}\right|$, establishing the lemma.

We now start with the construction of a selection from $F$. Let $\varepsilon>0$ and let $\mathscr{O}=\left\{O_{i} \mid i \in I\right\}$ be a uniform convex open cover of $X$ with sets of diameter $<\varepsilon$. Consider $S: Y \multimap|\mathscr{O}|$ as above and let $s$ be a selection of $S$ satisfying properties (1) and (2) of the lemma. Then $s$ can be decomposed into its affine coordinate functions $s_{i}: Y \rightarrow[0,1]$, which are all continuous. For each $y \in Y$ we put

$$
C_{i}(Y)= \begin{cases}O_{i}, & \text { if } s_{i}(y) \geq 1 / q+1 \\ X, & \text { otherwise }\end{cases}
$$

Consider a new multifunction defined as follows.

$$
G: Y \multimap X ; \quad G(y)=\mathrm{Cl}\left(\bigcap_{i \in I} C_{i}(y) \cap F(y)\right) .
$$

Note that $G(y)$ is nonempty since $s$ selects from $S$. In addition, the point $s(y)$ is in a simplex of $\mathscr{O}^{q}$, so one of its coordinates satisfies $s_{i}(y) \geq 1 / q+1$. This gives $C_{i}(y)=O_{i}$ and in particular $G(y) \subseteq \mathrm{Cl}\left(O_{i}\right)$ is of diameter $<\varepsilon$. We verify that $G$ is LSC at each point $y \in Y$. Let $P \subseteq X$ be an open set meeting $G(y)$, and fix a point $x \in P \cap \bigcap_{i \in I} C_{i}(y) \cap F(y)$. Each set $C_{i}(y)$ is open and 
all but finitely many are equal to $X$. Hence there is $\delta>0$ such that

$$
B(x, \delta)=\left\{x \mid d\left(x, x^{\prime}\right)<\delta\right\} \subseteq P \cap \bigcap_{i \in I} C_{i}(y)
$$

Consider a neighborhood $W_{1}$ of $y$ with the property

$$
\forall y^{\prime} \in W_{1}: F\left(y^{\prime}\right) \cap B(x, \delta) \neq \varnothing .
$$

Next, note that $s\left(W_{y}\right)$, being relatively compact, is included in a finite subcomplex of $|\mathscr{O}|$; cf. [46, p. 113]. This means that all but finitely many $s_{i}$ are identically zero on $W_{y}$, and consequently there is a neighborhood $W_{2} \subseteq W_{y}$ of $y$ such that if $i \in I$ and $s_{i}(y)<1 / q+1$ then $s_{i}\left(y^{\prime}\right)<1 / q+1$ for all $y^{\prime} \in W_{2}$. This readily implies that

$$
\forall y^{\prime} \in W_{2}, \quad \forall i \in I: C_{i}(y) \subseteq C_{i}\left(y^{\prime}\right) .
$$

If $y^{\prime} \in W_{1} \cap W_{2}$, then by (6) there is a point $x \in F\left(y^{\prime}\right) \cap B(x, \delta)$. By (5) and (7), we find that $x \in G\left(y^{\prime}\right)$.

Having completed the proof that $G$ is LSC, we apply the previous construction repeatedly to obtain a sequence $\left(F_{n}\right)_{n=0}^{\infty}$ of LSC multifunctions with convex closed values, such that

$$
F_{0}=F ; \quad F_{n}(y) \subseteq F_{n-1}(y) ; \quad \operatorname{diam} F_{n}(y)<1 / n .
$$

As $F(y)$ is complete, the set $\bigcap_{n=0}^{\infty} F_{n}(y)$ consists of one point $f(y)$ only. The resulting function $f: Y \rightarrow X$ selects from $F$ and is easily seen to be continuous.

The following observation due to Michael is of use in the proof of the general Selection Theorem. Let $\mathscr{X}$ be a family of subsets of a space $X$, including all singletons. Assume that every LSC multifunction $F: Y \rightarrow X$ with values in $\mathscr{X}$ admits a continuous selection. If $A \subseteq Y$ is closed, then each partial selection $f: A \rightarrow X$ of $F$ extends to a full selection of $F$. This follows from the fact that the multifunction $F^{\prime}$, defined by $F^{\prime}(y)=F(y)$ for $y \notin A$ and $F^{\prime}(y)=\{f(y)\}$ for $y \in A$, is LSC.

4.3. Theorem. Let $X$ be a topological $\mathrm{S}_{4}$ convex structure with compact polytopes and with connected convex sets, and let $d$ be a compatible metric on $X$.

(a) If $X$ is separable and if $Y$ is a $\mathrm{T}_{4}$ space, then each LSC multifunction $Y \multimap X$ with compact convex values admits a continuous selection.

(b) If $Y$ is a paracompact space, then each LSC multifunction $Y \multimap X$ with convex and $d$-complete values admits a continuous selection.

Proof. Let $\varepsilon>0$ and let $\delta>0$ be associated to $\varepsilon$ (as in 2.6). Let $F$ denote the given multivalued function. Take a locally finite open cover $\mathscr{P}$ refining the covering $\mathscr{B}_{\delta / 2}$ of all $\delta / 2$-balls in $X$, and let $\mathscr{V} \leq F^{-1}(\mathscr{P})$ be an open cover of $Y$ which will be specified later. Define a map $r_{0}:|\mathscr{V}|^{0} \rightarrow X$ as follows. For each $V \in \mathscr{V}$ select $P_{V} \in \mathscr{P}$ such that $V \subseteq F^{-1}\left(P_{V}\right)$, and choose a point $x_{V} \in P_{V}$. We put $r_{0}(V)=x_{V}$. Next, define a multivalued function as follows.

$$
R:|\mathscr{V}| \rightarrow X, R(u)=\operatorname{co}\left\{r_{0}(V) \mid V \in \sigma\right\},
$$

where $\sigma$ is the smallest simplex of $\mathscr{V}$ with $u \in|\sigma|$. This multifunction is easily seen to be LSC. If $r_{n}:|\mathscr{V}|^{n} \rightarrow X$ is a partial selection of $R$ over $|\mathscr{V}|^{n}$, and if $\sigma$ is an $(n+1)$-simplex of $|\mathscr{V}|$, then Proposition 4.2 and the remark preceding 
4.3 lead us to a partial selection of $R$ over $|\sigma|$ extending $r_{n}$. This yields a continuous extension $r_{n+1}$ of $r_{n}$ selecting from $R$ over $|\mathscr{V}|^{n+1}$. Proceeding by induction, we finally obtain a continuous selection $r:|\mathscr{V}| \rightarrow X$ of $R$. In each of the cases $(a),(b)$, we now specify the open cover $\mathscr{V}$.

Case (a). $X$ being separable, we may assume that $\mathscr{P}$ is countable moreover. As $F$ is compact-valued, it follows that $F^{-1}(\mathscr{P})$ is countable and point-finite. Hence $[19$, p. 394] there exists a (countable) star-finite open refinement $\mathscr{V}$ of $F^{-1}(\mathscr{P})$.

Case (b). As $Y$ is paracompact, $F^{-1}(\mathscr{P})$ admits a locally finite open refinement $\mathscr{V}$.

In both cases we infer that there exists a partition of unity $\left(g_{V}\right)_{V \in \mathscr{V}}$ subordinate to $\mathscr{V}$. This yields a map $g: Y \rightarrow|\mathscr{V}|$, and we show that

$$
\forall y \in Y: d(\operatorname{rg}(y), F(y))<\varepsilon .
$$

Let $\sigma=\left\{V_{0}, \ldots, V_{k}\right\}$ be the smallest simplex of $\mathscr{V}$ with $g(y) \in|\sigma|$. Then $g_{V_{i}}(y)>0$, so $y \in V_{i}$. Now $r_{0}\left(V_{i}\right)$ was chosen to be a point of some $P_{i} \in \mathscr{P}$ with $V_{i} \subseteq F^{-1}\left(P_{i}\right)$. Hence $P_{i}$ meets $F(y)$ for each $i$. As $\mathscr{P}$ refines $\mathscr{B}_{\delta / 2}$ and as $\delta$ is associated to $\varepsilon$, we find that

$$
\operatorname{rg}(y) \in \operatorname{co}\left\{r\left(V_{0}\right), \ldots, r\left(V_{k}\right)\right\} \subseteq \operatorname{co} B(F(y), \delta) \subseteq B(F(y), \varepsilon),
$$

establishing $(*)$.

Having shown that in each of the cases (a), (b), there is a "uniform approximation" of $F$ by maps $Y \rightarrow X$, we proceed as follows to obtain a selection. Let $n \in \mathbb{N}$, let $\varepsilon>0$ be associated to $1 / 2 n$, and let $g: Y \rightarrow X$ be $\varepsilon$-close to $F$. For each $y \in Y$, define

$$
G(y)=\mathrm{Cl}(\operatorname{co} F(y) \cap B(g(y), \varepsilon)) .
$$

Note that $G(y) \neq \varnothing$ and that $\operatorname{diam} G(y) \leq 1 / n$ since

$$
\operatorname{co} B(g(y), \varepsilon) \subseteq B(g(y), 1 / 2 n) .
$$

Let $O \subseteq X$ be an open set meeting $G(y)$ and fix a point

$$
x \in O \cap \operatorname{co}(F(y) \cap B(g(y), \varepsilon)) .
$$

By the third axiom of convexity there is a finite subcollection $\left\{x_{1}, \ldots, x_{k}\right\}$ of $F(y) \cap B(g(y), \varepsilon)$ with $x \in \operatorname{co}\left\{x_{1}, \ldots, x_{k}\right\}$. The operator co being LSC on $H_{\text {fin }}(X)$ (cf. 2.3), there is a $\delta>0$ with $\operatorname{co}\left\{x_{1}^{\prime}, \ldots, x_{k}^{\prime}\right\} \cap O \neq \varnothing$ for each $k$-tuple of points $x_{i}^{\prime} \in B\left(x_{i}, \delta\right)$. By taking a smaller $\delta$ if necessary, we may assume in addition that $d\left(g(y), x_{i}\right)+2 \delta<\varepsilon$ for all $i$. Consider a neighborhood $V$ of $y$ such that for all $y^{\prime} \in V$ and for each $i=1, \ldots, k$,

(i) $d\left(g\left(y^{\prime}\right), x_{i}\right)<d\left(g(y), x_{i}\right)+\delta$;

(ii) $F\left(y^{\prime}\right)$ meets the set $B\left(x_{i}, \delta\right)$.

If $y^{\prime} \in V$ then fix $x_{i}^{\prime} \in F\left(y^{\prime}\right) \cap B\left(x_{i}, \delta\right)$ for each $i=1, \ldots, k$. We find that

$$
d\left(g\left(y^{\prime}\right), x_{i}^{\prime}\right) \leq d\left(g\left(y^{\prime}\right), x_{i}\right)+d\left(x_{i}, x_{i}^{\prime}\right)<d\left(g(y), x_{i}\right)+2 \delta<\varepsilon .
$$

This yields

$$
\left\{x_{1}^{\prime}, \ldots, x_{k}^{\prime}\right\} \subseteq F\left(y^{\prime}\right) \cap B\left(g\left(y^{\prime}\right), \varepsilon\right) ; \quad \operatorname{co}\left\{x_{1}^{\prime}, \ldots, x_{k}^{\prime}\right\} \subseteq G\left(y^{\prime}\right),
$$


and since $x_{i}^{\prime} \in B\left(x_{i}, \delta\right)$ for each $i$, we also have $\operatorname{co}\left\{x_{1}^{\prime}, \ldots, x_{k}^{\prime}\right\} \cap O \neq \varnothing$. Consequently, $G\left(y^{\prime}\right)$ meets $O$ for each $y^{\prime} \in V$.

By inductive application of this procedure, we arrive at a sequence $\left(F_{n}\right)_{n \in \mathbb{N}}$ of LSC multifunctions $Y \multimap X$ with $F_{0}=F$ and $F_{n+1}(y)$ is a closed subset of $F_{n}(y)$ of diameter $<1 / n$ for each $n>0$ and $y \in Y$. There is only one point $f(y)$ in the set $\bigcap_{n=0}^{\infty} F_{n}(y)$, and the resulting function $f: Y \rightarrow X$ is clearly a continuous selection of $F$.

Two well-known selection theorems are plainly included in 4.3: the original Michael Theorem in Fréchet vector spaces, [32], and Nadler's result on selecting from subcontinua of a compact metric tree, [40]. Note that the restriction to compact spaces is, in fact, redundant.

\section{Absolute retracts, Hilbert CUbes, and dimension}

The main result in this section is the following.

5.1. Theorem. Let $X$ be a metrizable $\mathrm{S}_{4}$ convex structure with compact polytopes and with connected convex sets. Then each nonempty convex set in $X$ is an absolute retract.

Proof. Observe that the assumptions on $X$ are inherited by a convex subspace of $X$. We just show that $X$ is an absolute extensor (AE) for metric spaces. To this end, let $A$ be a closed subset of a metric space $Y$ and let $g: A \rightarrow X$ be a mapping. We use a technique of Dugundji [13] to extend $g$ over $Y$. Let $d(y, A)$ denote the distance of $y$ to $A$. The covering $\{B(y, d(y, A)) \mid y \in$ $Y \backslash A\}$ of $Y \backslash A$ admits a locally finite open refinement $\mathscr{U}$. For each $U \in \mathscr{U}$ we fix $p_{U} \in \mathscr{U}$ and we choose $a_{U} \in A$ in such a way that $d\left(p_{U}, a_{U}\right)<$ $2 \cdot d\left(p_{U}, A\right)$. Consider a multifunction $F: Y \rightarrow X$ defined as follows.

$$
F(y)= \begin{cases}\{g(y)\}, & \text { if } y \in A ; \\ \operatorname{co}\left\{a_{U} \mid y \in U \in \mathscr{U}\right\}, & \text { otherwise. }\end{cases}
$$

If $y \in Y \backslash A$, then $\bigcap\{U \mid y \in U \in \mathscr{U}\}$ is a neighborhood of $y$ such that for each element $y^{\prime}$ of it, $F\left(y^{\prime}\right) \supseteq F(y)$. Consequently, $F$ is LSC. To see that $F$ is even continuous at $a \in A$, consider a convex neighborhood of $g(y)$ in $X$ and argue as in [13, p. 358].

By Theorem 4.3, $F$ has a continuous selection, which necessarily extends $g$ over $Y$.

Each of the following spaces (satisfying the conditions of the last theorem) are known to be absolute retracts: convex sets in metric locally convex vector spaces [13, 4.2]; metric trees [5, 13.5]; and certain classes of compact metric (semi-)lattices (folklore; these spaces are natural retracts of their hyperspace). For spaces of order arcs (with given endpoints), selection seems to be the only reasonable way to obtain the AR-property. For convex hyperspaces, the ARproperty is known in traditional cases: vector spaces, and compact trees (where the convex hyperspace reduces to the hyperspace of subcontinua). See 5.4 for new examples.

We finally note that each AR carries a Michael convexity, but a convex structure as in 5.1 requires a space which is both globally and locally an AR (by local convexity). 
Theorems 4.1 and 5.1 have lead to various results on coinciding dimension functions. For instance, in [58] it is shown that $\operatorname{dim}=$ ind $=\operatorname{Ind}=\mathrm{cd}^{1}$ on a class of arcwise connected Lawson semilattices. In completely distributive lattices, the condition on arcwise connectedness can be dropped; cf. [56]. Finally, [39] contains a result on compact spaces with a uniform convexity. Its proof involves an application of Theorem 5.2 below.

Recall [53] or [51] that the convex dimension, $\operatorname{cind}(X)$, of a topological convex structure $X$ is defined by the following two rules, where $-1 \leq n<\infty$.

(1) $\operatorname{cind}(X)=-1$, iff $X=\varnothing$.

(2) $\operatorname{cind}(X) \leq n+1$ iff for each convex closed set $C \subseteq X$ and for each point $p \notin C$ there exist convex closed sets $A, B$ covering $X$, such that

$$
C \cap B=\varnothing ; \quad p \notin A ; \quad \operatorname{cind}(A \cap B) \leq n .
$$

This dimension function was studied in [51], where it was shown that cind of a topological convex structure equals the Lebesgue dimension of the underlying space provided the latter is separable and metrizable, in addition to the following requirements on the convexity.

(i) Closure stability.

(ii) A convex closed set and a point outside are separated by a real-valued CP map.

(iii) All convex sets are weakly homotopy trivial.

Note that the first and second condition are fulfilled on a uniform $\mathrm{S}_{4}$ convex structure by the results of $\S 2$. The third is a consequence of Theorem 5.1. In conclusion, we have

5.2. Theorem. If $X$ is a separable, metrizable, $\mathrm{S}_{4}$ convex structure with connected convex sets and with compact polytopes, then $\operatorname{cind}(X)=\operatorname{dim}(X)$.

The elimination of weak homotopy triviality (cf. condition (iii) above) in favour of more manageable conditions, like connectedness of convex sets and uniform continuity of the hull operator, has been one of our motives to search for a selection theorem.

Recall [43] that a collection $\mathscr{A}$ of sets in a metric space is equi-uniformly Lefschetz provided for each $\varepsilon>0$ there exists a $\delta>0$ with the property that every partial realization of norm $<\delta$ of a polyhedron into a set $A \in \mathscr{A}$, extends to a full realization of norm $<\varepsilon$ into $A$.

5.3. Corollary. If $X$ is a metrizable $\mathrm{S}_{4}$ convex system with connected convex sets and with compact polytopes, then the convexity of $X$ is an equi-uniformly Lefschetz family.

Proof. Fix $\varepsilon>0$ and consider $\delta>0$ such that admissible sets of diameter $<\delta$ have a convex hull of diameter $<\varepsilon$. Consider a polyhedron $P$ with a fixed triangulation. Let $P_{0} \subseteq P$ be a subpolyhedron including the 0 -skeleton $P^{0}$, and let $f_{0}: P_{0} \rightarrow C$ be a map into a convex set $C$ of $X$. We assume $f_{0}$ is of norm $<\delta$, that is

$$
\operatorname{diam} f_{0}\left(|\sigma| \cap P_{0}\right)<\delta
$$

\footnotetext{
${ }^{1} \mathrm{~cd}$ : cohomological dimension; $\mathrm{cf}$. Cohen [9].
} 
for each simplex $\sigma$ of $P$. Note that the convex set $C(\sigma)=$ co $f_{0}\left(|\sigma| \cap P_{0}\right)$ is included in $C$ and has diameter $<\varepsilon$. After applying Theorem 5.1 to the 1skeleton of each simplex of $P$, we obtain an extension of $f_{0}$ to a map $f_{1}: P_{1}=$ $P_{0} \cup P^{1} \rightarrow C$, with $f_{1}\left(|\sigma| \cap P_{1}\right) \subseteq C(\sigma)$ for each simplex $\sigma$ of $P$. Having obtained a mapping

$$
f_{i}: P_{i}=P_{i-1} \cup P^{i} \rightarrow C,
$$

such that $f_{i}\left(|\sigma| \cap P_{i}\right) \subseteq C(\sigma)$ for each simplex $\sigma$ of $P$, application of Theorem 5.1 to the $(i+1)$-skeleton of each simplex yields an extension $f_{i+1}$ satisfying (\#) with $i+1$ replacing $i$. This finally leads to an extension $f: P \rightarrow X$ of $f_{0}$ ranging into $C$ and such that $f(|\sigma|) \subseteq C(\sigma)$ for each simplex $\sigma$ of $P$. In particular, $f$ is of norm $<\varepsilon$.

Pixley [43] raised the question of whether an LSC multifunction, taking values in an equi-uniformly Lefschetz family, admits a continuous selection. If the domain is finite dimensional, then the answer is affirmative by Michael's results of [33]. In particular cases, such as the system of convex sets of the EberhartNadler-Nowell space (cf. $\S 3$ ), the answer is also affirmative since the convex system extends to a convex structure as required in the Selection Theorem 4.3. This fact is used in our next result.

Recall (Ward [66]) that a Whitney map on a partially ordered space $Y$ is a map $w: Y \rightarrow[0,1]$ with the following properties. mal.

(i) (Normalization) $w(y)=0$ for $y$ minimal and $w(y)=1$ for $y$ maxi-

(ii) (Isotony) If $y_{1}<y_{2}$, then $w\left(y_{1}\right)<w\left(y_{2}\right)$.

Such functions are usually considered on hyperspaces of compact (connected) sets. See Nadler [40]. In general, sets of type $w^{-1}(t)$ are called Whitney levels. Petrus [42] has shown that a Whitney level of subcontinua need not be an AR, even if the basic space is. On the other hand, she showed that Whitney levels of subcontinua of a compact tree must be AR's. The following corollary (second part) extends this result.

5.4. Corollary. Let $X$ be a compact metrizable $\mathrm{S}_{4}$ convex structure with connected convex sets. Then the following hold.

(1) The space of all maximal arcs of convex closed sets is an AR.

(2) Each Whitney level of the convex hyperspace is an AR.

Proof of (1). Let $\mathscr{C}$ denote the convexity of $X$. As usual, $C(X, \mathscr{C})$ denotes the corresponding convex hyperspace and $\Lambda C(X, \mathscr{C})$ is the corresponding space of maximal arcs. The hyperspace of subcontinua of $X$ is $C(X)$, and then $\Gamma C(X)$ is the Eberhart-Nadler-Nowell space of all arcs in $C(X)$. It is shown in [16] that $\Gamma C(X)$ is an $\mathrm{AR}$. We construct a retraction $\Gamma C(X) \rightarrow \Lambda C(X, \mathscr{C})$ as follows. First, an arc $\alpha$ in $C(X)$ is replaced with the corresponding arc $\alpha^{\prime}$ of convex closures. Then we attach two continuously selected arcs of convex closed sets, one joining some singleton to the initial element of $\alpha^{\prime}$, the other one joining the final element of $\alpha^{\prime}$ to $X$.

As for the first process, consider a continuous selection

$$
g: C(X, \mathscr{C}) \rightarrow X,
$$


that is: $g(C) \in C$ for all $C$. Then consider the multivalued function

$$
G: C(X, \mathscr{C}) \multimap \Gamma C(X), \quad G(C)=\{\beta \mid \beta \text { joins } g(C), C\} .
$$

A straightforward proof, that $G$ is LSC and has compact convex values, is left to the reader. Select continuously from $G$, and replace the resulting arcs by the corresponding arcs of convex closures. This describes the "prepending" process.

Secondly, consider the LSC multifunction

$$
H: C(X, \mathscr{C}) \multimap \Gamma C(X), \quad H(C)=\{\gamma \mid \gamma \text { joins } C, X\},
$$

which has compact and convex value sets. A continuously selected arc is again replaced with the arc of convex closures, and is used in the "appending" process.

This completes the description of a retraction and establishes (1).

Proof of 2. Let $w: C(X, \mathscr{C}) \rightarrow[0,1]$ be a Whitney map and let $0 \leq t \leq 1$. The multifunction

$$
F: w^{-1}(t) \multimap \Lambda(X), \quad F(C)=\{\alpha \mid \alpha \text { contains } g(C), C\}
$$

(where $g$ is the mapping described in (3) above) is LSC and has compact convex value sets. We extract a continuous selection of it, and we transform each arc into the corresponding arc of convex closures. Since the elements of $w^{-1}(t)$ are mutually incomparable, we obtain an embedding of this compact space into $\Lambda C(X, \mathscr{C})$. On the other hand, there is an "evaluation" map

$$
e_{t}: \Lambda C(X, \mathscr{C}) \rightarrow w^{-1}(t),
$$

sending an arc to its one-and-only member at the $t$-level. This map retracts $\Lambda C(X, \mathscr{C})$ back onto $w^{-1}(t)$. By the first part of the Corollary, $w^{-1}(t)$ is an AR.

Remark. ${ }^{2}$ For a space $X$ as above, one can easily verify that the endpoint map $e: \Lambda C(X, \mathscr{C}) \rightarrow X$, sending a maximal arc in $C(X, \mathscr{C})$ to its (singleton) end, is open. For each $x \in X$, the fiber $e^{-1}(x)$ is a convex subspace of $\Lambda C(X, \mathscr{C})$, and hence it is an AR by Theorem 5.1. One might think this is far enough to conclude that $\Lambda C(X, \mathscr{C})$ is an AR. It is not. Taylor [47] constructed a cell-like surjection of a space onto the Hilbert cube $Q$ which is not a shape equivalence. By a result of Kozlowski, van Mill, and Walsh [28], such a map can be transformed into a mapping $Z \rightarrow Q$ which is an open retraction with AR fibers, and which is not a hereditary shape equivalence. By a result of Kozlowski [27, Theorem 10], its domain $Z$ cannot be an AR.

We proved in $[53,6.12]$ that if $X$ is a finite dimensional convex structure with connected convex sets, then the intersection of all dense convex subsets of $X$ is dense. In particular, every two dense convex sets intersect. Hence this property must also hold in spaces with a nonempty, convex, open, and finite dimensional subspace, that is: in spaces which are "somewhere finite dimensional." This observation may contribute to the appreciation of the following result.

5.5. Theorem. Let $X$ be a (locally) compact metrizable $\mathrm{S}_{4}$ convex structure with connected convex sets and with compact polytopes. If every pair of polytopes on $X$ can be approximated by a pair of disjoint polytopes-in particular, if there

\footnotetext{
${ }^{2}$ I am indebted to Jan van Mill for this observation.
} 
is a pair of disjoint, dense, and convex subsets in $X$-then $X$ is a Hilbert cube (manifold).

Proof. As $X$ is an AR in Theorem 5.1, by a result of Torunczyk [50, p. 36] it suffices to show that each pair of mappings $f, g: Q \rightarrow X$ (where $Q$ denotes the Hilbert cube) can be approximated with mappings having disjoint images. To this end, let $\mathscr{O}$ be a convex open cover of $X$, and consider a barycentric refinement $\mathscr{V}$ of some star refinement of $\mathscr{O}$. Furthermore, let $\mathscr{W}$ be a finite open cover of $Q$ refining both $f^{-1}(\mathscr{V})$ and $g^{-1}(\mathscr{V})$. Choose a point $t_{W} \in W$ for each $W \in \mathscr{W}$ and consider the following polytopes.

$$
P(f)=\operatorname{co}\left\{f\left(t_{W}\right) \mid W \in \mathscr{W}\right\} ; \quad P(g)=\operatorname{co}\left\{g\left(t_{W}\right) \mid W \in \mathscr{W}\right\} .
$$

By assumption, we obtain a pair of disjoint polytopes $P^{\prime}(f), P^{\prime}(g)$ which are $\mathscr{V}$-close to $P(f), P(g)$, respectively. For each $W \in \mathscr{W}$ we fix two points as follows: $y_{W} \in P^{\prime}(f)$ is $\mathscr{V}$-close to $f\left(t_{W}\right)$, and $z_{W} \in P^{\prime}(g)$ is $\mathscr{V}$-close to $g\left(t_{W}\right)$. This leads to the following LSC polytope-valued multifunctions.

$$
F: Q \rightarrow X: F(t)=\operatorname{co}\left\{y_{W} \mid t \in W\right\}, \quad G: Q \rightarrow X: G(t)=\operatorname{co}\left\{z_{W} \mid t \in W\right\} .
$$

Take continuous selections $f^{\prime}, g^{\prime}$, respectively. For $t \in Q$ we see that the points $y_{W}$ with $W \ni t$ are in the set $\operatorname{st}(\operatorname{st}(f(t), \mathscr{V}) \mathscr{V})$, which in turn is included in some $O \in \mathscr{O}$. As $O$ is convex, we find that $F(t) \subseteq O$ and, consequently, that $f(t)$ and $f^{\prime}(t)$ are $\mathscr{O}$-close. Similarly, $g(t)$ is $\mathscr{O}$-close to $g^{\prime}(t)$. The images of $f^{\prime}, g^{\prime}$ are evidently disjoint.

The simplest case where this result applies is the following. Let $C$ be a compact infinite dimensional convex set of a topological vector space. Elementary considerations yield that every pair of polytopes can be approximated with a pair of disjoint polytopes. Hence, if $C$ is metrizable and locally convex, then $C$ is a Hilbert cube. This result is known as Keller's Theorem, [4, p. 100, 36, p. 378].

For a quick second example, consider the superextension of a nontrivial metric continuum $X$. The resulting topological convex structure is compact by construction, connected by [63, p. 99], and (topologically) metrizable by [63, p. 107]. Its convex sets are connected (cf. 3.3), and here is a method to produce two disjoint dense convex subsets. Elementary topological considerations yield two disjoint dense subsets $A_{1}, A_{2}$ of the original space $X$. Then the desired convex sets are (with the notation of 3.3):

$$
C_{i}=\bigcup\left\{F^{+} \mid F \subseteq A_{i}, F \text { finite }\right\} \quad(i=1,2) .
$$

In [59] we have obtained a much stronger result stating that a convex closed subset of a superextension (as above) is either a one-point set or is a topological copy of the Hilbert cube. The lengthy proof is based again on the Selection Theorem but, in addition, it requires more specific results from infinite dimensional topology.

For a final application, we consider the space $\Lambda(S)$ of all maximal order arcs in an arcwise connected Lawson semilattice $S$. For instance, if $X$ is a continuum and if $p \in X$, then $S$ can be taken as the hyperspace of all subcontinua of $X$ containing $p$. 


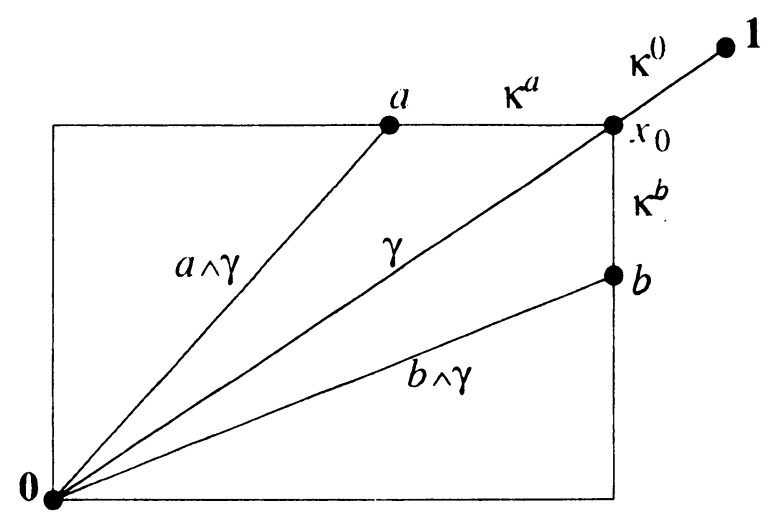

FIGURE 3. Arc modification

5.6. Theorem. Let $S$ be an arcwise connected metric Lawson semilattice with a top element. If $S$ is not reduced to an arc, then $\Lambda(S)$ is homeomorphic to the Hilbert cube.

Proof. If $S$ is not an arc, then there is a point of $S$ each neighborhood of which has incomparable elements. There clearly is a largest point $x_{0}$ with this property. Note that each arc joining the bottom element 0 with the top element 1 passes through $x_{0}$, and that the upper set $\geq\left(x_{0}\right)$ is a (possibly degenerate) arc $\kappa^{0}$. See Figure 3.

Let $\delta>0$ and take $\rho>0$ such that $\rho$-close pairs of points have $\delta$-close infima. Consider two incomparable points $a, b$ in some order-convex neighborhood $N$ of $x_{0}$ of diameter $<\delta$. Let $\kappa^{a}, \kappa^{b}$ be two arcs joining $x_{0}$ with $a, b$, respectively. Being included in $N$, these arcs have diameter $<\delta$.

If $\gamma$ is an arc joining 0 to 1 , then define $\gamma^{a}=(a \wedge \gamma) \cup \kappa^{a} \cup \kappa^{0}$. This yields a new arc joining 0 to 1 . As $a, x_{0}$ are $\rho$-close, the sets $a \wedge \gamma, x_{0} \wedge \gamma$ are $\delta$-close. Note that the second arc is the part of $\gamma$ from $\mathbf{0}$ up to $x_{0}$. Furthermore, $\gamma$ and $\gamma^{a}$ share the arc $\kappa^{0}$. As $\operatorname{diam} \kappa^{a}<\delta$, we conclude that $\gamma$ and $\gamma^{a}$ are $\delta$-close.

In a similar way, we can modify $\gamma$ into an arc $\gamma^{b}=(b \wedge \gamma) \cup \kappa^{b} \cup \kappa^{0}$ joining $\mathbf{0}, \mathbf{1}$, and which is $\delta$-close to $\gamma$.

This is used to approximate two polytopes $P=\operatorname{co}\left\{\alpha_{1}, \ldots, \alpha_{n}\right\} ; Q=$ $\operatorname{co}\left\{\beta_{1}, \ldots, \beta_{m}\right\}$ in $\Lambda(S)$ with disjoint polytopes as follows. Let $\varepsilon>0$ and let $\delta>0$ be such that $\delta$-close sets in $\Lambda(S)$ have $\varepsilon$-close hulls. The above procedure leads us to two polytopes of type

$$
P^{a}=\operatorname{co}\left\{\alpha_{1}^{a}, \ldots, \alpha_{n}^{a}\right\} ; \quad Q^{b}=\operatorname{co}\left\{\beta_{1}^{b}, \ldots, \beta_{m}^{b}\right\},
$$

which are $\varepsilon$-close to the respective originals. Now each member of the first is an arc through $a$, whereas each member of the second passes through $b$. Therefore, the approximating polytopes are disjoint

By way of example, a continuum $X$ is indecomposable at $p \in X$ provided all subcontinua containing $p$ are mutually comparable. Note that $X$ is hereditarily indecomposable, [40, p. 17], iff it is indecomposable at each point. If a metric continuum is decomposable at $p$, then the space of all maximal arcs of continua ending at $p$ is a Hilbert cube. 


\section{APPROXIMATION AND FIXED POINTS}

We first work towards a result of G. Beer [2, Theorem 1] on approximating USC convex-valued functions by singlevalued ones. Weaker results were obtained earlier by Cellina (cf. $[7,8])$. We do not only generalize these results to nonvector spaces, but we also give a simpler proof based on two ordinary selection procedures.

Recall that an enlargement of a multifunction is one with larger value sets. If $X, Y$ are metric spaces, then a USC closed-valued function $Y \multimap X$ has a closed graph in $Y \times X$, and it makes sense to consider the Hausdorff distance between such functions $(Y \times X$ with Cartesian metric).

6.1. Lemma. Let $X$ be a complete, metric, $\mathrm{S}_{4}$ convex structure with compact polytopes and with connected convex sets, and let $F: Y \rightarrow X$ be a USC function with compact convex values. Then for each $\varepsilon>0$ there is a continuous enlargement of $F$ with compact convex value sets, and which is $\varepsilon$-close to $F$.

Proof. Let $\mathscr{C}$ denote the convexity of $X$ and let $d$ be the complete compatible metric. The convex hyperspace is convexified with the "Vietoris" point of view; see 3.7. It inherits the properties $\mathrm{S}_{4}$, compactness of polytopes, connectedness of all convex sets, and metrizability. In fact, the Hausdorff metric induced by $d$ is compatible with the Vietoris convexity, $[57,1.4]$. Note that this metric is complete on the hyperspace of all compacta, [19], and hence it is complete on the closed subspace $C(X, \mathscr{C})$, consisting of all compact convex sets. Hence, $C(X, \mathscr{C})$ is completely metrizable as a topological convex structure.

First, consider the following multifunction.

$$
\mathscr{F}: Y \multimap C(X, \mathscr{C}) ; \quad \mathscr{F}(y)=\{C \mid F(y) \subseteq C \in C(X, \mathscr{C})\} .
$$

Clearly, the collection $\mathscr{F}(y)$ is convex closed. To see that $\mathscr{F}$ is LSC at $y \in Y$, consider a basic open set $\left\langle O_{1}, \ldots, O_{n}\right\rangle$ (notation of 1.4) of the hyperspace, and let $C \in\left\langle O_{1}, \ldots, O_{n}\right\rangle \cap \mathscr{F}(y)$. Then $C \subseteq \bigcup_{i=1}^{n} O_{i}$ and by Theorem 2.6, there is a convex open set $O \subseteq X$ with $F(y) \subseteq C \subseteq O \subseteq \bar{O} \subseteq \bigcup_{i} O_{i}$. As $\mathscr{F}$ is USC, we find a neighborhood $W$ of $y$ with $F\left(y^{\prime}\right) \subseteq O$ whenever $y^{\prime} \in W$. choose $x_{i} \in C \cap O_{i}$ for $i=1, \ldots, n$, and consider $y^{\prime} \in W$. The set

$$
C^{\prime}=\mathrm{Cl} \operatorname{co}\left(F\left(y^{\prime}\right) \cup\left\{x_{1}, \ldots, x_{n}\right\}\right)
$$

is compact by Theorem 2.8. As $\bar{O}$ is convex, we find that $C^{\prime} \subseteq \bar{O}$, whence $C^{\prime} \in \mathscr{F}\left(y^{\prime}\right) \cap\left\langle O_{1}, \ldots, O_{n}\right\rangle$.

Note that a continuous selection of $\mathscr{F}$ is just a continuous enlargement of $F$ with compact convex value sets. To complete the proof of the lemma, we restrict the "choice margins" of $\mathscr{F}$ such that any continuous selection of the shrunken map is as required. To this end, let $\delta>0$ be such that $\delta$-close subsets of $X$ have $\varepsilon / 2$-close hulls. For each $y \in Y$ pick a neighborhood $U_{y}$ such that

$$
\begin{gathered}
\operatorname{diam} U_{y}<\varepsilon . \\
\forall y^{\prime} \in U_{y}: F\left(y^{\prime}\right) \subseteq B(F(y), \delta) .
\end{gathered}
$$

Choose a locally finite open refinement $\mathscr{V}$ of $\left\{U_{y} \mid y \in Y\right\}$, and fix a closed shrinking $\mathscr{D}=\left\{D_{V} \mid V \in \mathscr{V}\right\}$ of $\mathscr{V}$, that is: $D_{V}$ is a closed set included in 
$V$ for all $V \in \mathscr{V}$. Such covers exist, [14, VII.6.1]. For $y \in Y$ we obtain a neighborhood

$$
W(y)=\bigcap\{V \mid y \in V \in \mathscr{V}\} \backslash \bigcup\left\{D_{V} \mid y \notin D_{V}\right\} .
$$

Finally, for each $V \in \mathscr{V}$ we fix a point $p(V)$ with $V \subseteq U_{p(V)}$. Consider the set

$$
C_{V}(y)= \begin{cases}B(F(p(V)), \delta), & \text { if } y \in D_{V} \\ X, & \text { otherwise. }\end{cases}
$$

By (3), we find $C_{V}(y) \subseteq C_{V}\left(y^{\prime}\right)$ for $y^{\prime} \in W(y)$. This readily implies lower semicontinuity of the multifunction $\mathscr{G}: Y \rightarrow C(X, \mathscr{C})$, defined by the prescription

$$
\begin{aligned}
\mathscr{G}(y) & =\mathrm{Cl}\left(\operatorname{co}\left(\bigcap_{V \in \mathscr{V}}\left\langle C_{V}(y)\right\rangle \cap \mathscr{F}(y)\right)\right) \\
& =\mathrm{Cl}\left(\operatorname{co}\left\{C \mid F(y) \subseteq C \in C(X, \mathscr{C}) ; \forall V \in \mathscr{V}: C \subseteq C_{V}(y)\right\}\right)
\end{aligned}
$$

(convex hull taken in the convex hyperspace). The set $\mathscr{G}(y)$ is evidently closed and convex. By virtue of (2), it contains the "element" $F(y)$. Extract a continuous selection $G$ from $\mathscr{G}$. We must verify that if $x \in G(y)$, then $(y, x)$ is $\varepsilon$ close to some point of (the graph of) $F$. Let $V \in \mathscr{V}$ be such that $y \in D_{V}$, and consider the corresponding point $p(V)$ with $V \subseteq U_{p(V)}$. Then $d(y, p(V))<\varepsilon$ by (1), whereas by (3),

$$
\begin{aligned}
G(y) & \subseteq \mathrm{Cl}\left(\operatorname{co} C_{V(y)}\right)=\mathrm{Cl}(\operatorname{co} B(F(p(V)), \delta)) \\
& \subseteq \mathrm{Cl}(B(F(p(V)), \varepsilon / 2)) \subseteq B(F(p(V)), \varepsilon) .
\end{aligned}
$$

Hence, $x$ is $\varepsilon$-close to some point of $F(p(V))$, establishing the result.

A result similar to Lemma 6.1 has been obtained by Aseev [6] for the standard convexity of $X=\mathbb{R}^{n}$. We now present the second announced selection procedure.

6.2. Lemma. Let $X$ be a metric $\mathrm{S}_{4}$ convex structure with connected convex sets and with compact polytopes, and let $Y$ be a metric space without isolated points. If $G: Y \rightarrow X$ is continuous and has compact convex values, then for each $\varepsilon>0$ there is a continuous (single-valued) selection of $G$, the graph of which is $\varepsilon$-close to $G$.

Proof. Let $\mathscr{U}$ be a locally finite open cover of $X$ with sets of diameter $<\varepsilon$. For each $U \in \mathscr{U}$ let

$$
V(U)=\{y \mid G(y) \cap U \neq \varnothing\} .
$$

This set is open since $G$ is LSC. For each $y \in Y$ there is an open set $O \subseteq$ $X$ such that $G(y) \subseteq O$ and $O$ meets only finitely many $U \in \mathscr{U}$. As $G$ is USC, there is a neighborhood $W(y)$ of $y$ such that $G\left(y^{\prime}\right) \subseteq O$ whenever $y^{\prime} \in W(y)$. In particular, $W(y)$ meets only finitely many of the sets $V(U)$, $U \in \mathscr{U}$, showing that the corresponding cover of $Y$ is locally finite.

For each $U \in \mathscr{U}$ we fix a closed discrete set $S(U) \subseteq V(U)$ which is $\varepsilon$-close to $V(U)$. As $Y$ has no isolated points and as the open sets $V(U)$ constitute a locally finite collection, care can be taken that $S(U) \cap S\left(U^{\prime}\right)=\varnothing$ for $U \neq U^{\prime}$. The set $A=\bigcup_{U \in \mathscr{U}} S(U)$ yields a closed and discrete subspace of $Y$, and 
a continuous partial selection $g: A \rightarrow X$ of $G$ is obtained as follows. For $a \in A$, say: $a \in S(U)$, choose $g(a) \in G(a) \cap U$. Any extension of $g$ to a full continuous selection of $G$ is $\varepsilon$-close to the graph of $G$.

6.3. Theorem. Let $X$ be a metric $\mathrm{S}_{4}$ convex structure with connected convex sets and with compact polytopes. Let $Y$ be a metric space without isolated points, and let $F: Y \multimap X$ be a USC, compact convex-valued function. Then for each $\varepsilon>0$ there is a map $Y \rightarrow X$ the graph of which is $\varepsilon$-close to $F$.

Proof. According to [52, 2.6, 2.7], $X$ extends to a complete metric convex structure $X^{*}$ inheriting all properties of $X$ currently considered. Moreover, $X$ is a dense convex subspace of $X^{*}$. Put $F^{*}(y)=\mathrm{Cl} F(y)$ (closure in $X^{*}$ ) and enlarge $F^{*}$ to a continuous multifunction $G: Y \multimap X^{*}$ (as in 6.1), which is $\varepsilon / 3$-close to $F^{*}$. Next, select a map $g: Y \rightarrow X$ from $G$ having its graph at a distance less than $\varepsilon / 3$ away from $G$. Finally, a simple application of the selection theorem yields a map $f: Y \rightarrow X \subseteq X^{*}$ which is $\varepsilon / 3$-close to $g$ (even in the sup-metric). Then $f$ is the desired approximation to $F$.

Our next goal is to establish a semilattice-theoretic counterpart of Theorem 6.1 which is based on the natural concepts of upper- and lower semicontinuity of functions into certain semilattices.

6.4. Continuous semilattices. Let $S$ be a semilattice, and let $x, y \in S$. Then $x$ is way-below $y$-in symbols: $x \ll y$-provided for each up-directed set $D \subseteq S$ with $\sup D \geq y$ it is true that $d \geq x$ for some $d \in D$. A complete semilattice $S$ is continuous provided each $y \in S$ is the supremum of all $x \in S$ with $x \ll y$. We quote two elementary facts for later use. See [20, I.1].

(1) If $x_{i} \ll y$ for $i=1,2$, then $\sup \left\{x_{1}, x_{2}\right\} \ll y$.

(2) If $x \ll z$ then there exists a point $y$ with $x \ll y \ll z$ (interpolation property).

The fundamental theorem of continuous semilattices states that the complete continuous semilattices are exactly the ones admitting a compatible, compact, Hausdorff, and locally convex topology. See [20, VI.3.4]. This combines with a result of Lawson, [29, Corollary 16]: a semilattice admits at most one compatible, compact Hausdorff topology. For a continuous semilattice $S$, this topology is generated by the open sets of type

$$
\begin{array}{ll}
\gg(x)=\{y \mid x \ll y\}, & x \in S ; \\
S \backslash \geq(x)=\{y \mid x \not \leq y\}, & x \in S .
\end{array}
$$

If $Y$ is a space and if $S$ is a continuous semilattice, then a function $Y \rightarrow S$ is lsc (resp. usc) provided it inverts the sets of type (3) (resp., (4)) into open sets of $Y$. It is easily verified that $f: Y \rightarrow S$ is lsc iff its upper graph $\{(y, s) \mid s \geq f(y)\}$ is closed, and that $f$ is usc iff its lower graph (defined similarly) is closed.

A classical example of a continuous semilattice is the hyperspace $H(X)$ of a compact space $X$. The partial order is reverse inclusion (containment), and the intrinsic topology is the Vietoris topology, [20, VI.3.8]. As a consequence of inverting the inclusion-order in $H(X)$, an LSC (USC) closed-valued function corresponds to a usc (lsc) function into the semilattice $H(X)$. In the sequel, we will specify the viewpoint.

Let $[Y, S]$ denote the collection of all lsc functions $Y \rightarrow S$. Note that this set is a semilattice under "pointwise" ordering of functions. The infimum of 
infinite families may exist, but is usually not the pointwise infimum. In [20, pp. 137-138] it is shown that if $Y$ is locally compact, then $[Y, S]$ is complete and continuous semilattice. For a compact space $Y$, this can also be seen from the fact that $[Y, S]$ is isomorphic to the hyperspace of all upper closed sets in $Y \times S$. The latter are sets $A$ projecting onto $Y$, and such that $(y, s) \in A$ and $s \leq s^{\prime}$ imply $\left(y, s^{\prime}\right) \in A$. The semilattice operation is the union operation.

6.5. Theorem. Let $S$ be an arcwise connected metrizable Lawson semilattice, the intervals of which depend continuously on their endpoints. Let $Y$ be a locally compact space.

(a) If $f_{0}, f_{1}: Y \rightarrow S$ are lsc functions such that $f_{0} \ll f_{1}$, then there exists a continuous function $f: Y \rightarrow S$ with $f_{0} \leq f \ll f_{1}$.

(b) Each lsc function $Y \rightarrow S$ is the supremum of an updirected family of continuous functions $Y \rightarrow S$.

(c) If $Y$ is separable and metrizable, then each lsc function $Y \rightarrow S$ is the supremum of an increasing sequence of continuous functions $Y \rightarrow S$.

Proof. The way-below relation in $[Y, S]$ can be described as follows. Let $f_{0}, f_{1} \in[Y, S]$. Then $f_{0} \ll f_{1}$ iff there exist open sets $U_{i}, V_{i} \subseteq Y$ such that $U_{i}^{\prime} \subseteq V_{i}, \bigcup_{i=1}^{n} U_{i}=Y$, together with points $s_{i} \ll t_{i}$ of $S$, such that for all $i$,

$$
y \in U_{i}^{-} \Rightarrow f_{0}(y) \leq s_{i} ; \quad y \in V_{i}^{-} \Rightarrow t_{i} \leq f_{1}(y) .
$$

Consider a multifunction $F: Y \multimap S$, defined by

$$
F(y)=\left[\sup \left\{s_{i} \mid y \in U_{i}^{-}\right\}, \sup \left\{t_{i} \mid y \in V_{i}\right\}\right] .
$$

Note that $F(y)$ is nonempty and that $F\left(y^{\prime}\right) \supseteq F(y)$ whenever $y^{\prime}$ is in the $y$-neighborhood

$$
\bigcap_{i=1}^{n}\left\{V_{i} \mid y \in V_{i}\right\} \backslash \bigcup_{i=1}^{n}\left\{U_{i}^{-} \mid y \notin U_{i}^{-}\right\} .
$$

So $F$ is LSC (as a multifunction). Let $f$ be a continuous selection of $F$. If $y \in U_{i}$ then

$$
f_{0}(y) \leq \sup \left\{s_{i} \mid y \in U_{i}^{-}\right\} \leq f(y) .
$$

On the other hand, let $i_{1}, \ldots, i_{k}$ be all values of $i$ for which $y \in V_{i}$. Then

$$
f(y) \leq \sup \left\{t_{i} \mid y \in V_{i}\right\} \leq f_{1}(y),
$$

and it follows that $f \leq f_{1}$.

We prove (a) as follows. As $[Y, S]$ is a continuous semilattice, the interpolation property $6.4(2)$ holds: there is a $f_{1}^{\prime} \in[Y, S]$ with $f_{0} \ll f_{1}^{\prime} \ll f_{1}$. By the above argument, there is a continuous $f: Y \rightarrow S$ with $f_{0} \leq f \leq f_{1}^{\prime} \ll f_{1}$.

As for (b), let $g: Y \rightarrow S$ be lsc. If $f_{1}, \ldots, f_{n}$ are continuous functions $Y \rightarrow S$ with $f_{i} \ll g$ for all $i$, then $\sup \left\{f_{1}, \ldots, f_{n}\right\} \ll g$. See 6.4(1). Note that the map on the left is lsc, but not necessarily continuous. By (a), however, there is a continuous $f: Y \rightarrow S$ with

$$
\sup \left\{f_{1}, \ldots, f_{n}\right\} \leq f \ll g .
$$

To obtain (c), we use $[20$, p. 172]: the weight of the space $[Y, S]$ is the maximum of weight $(Y)$ and weight $(S)$. Hence $[Y, S]$ is metrizable if $Y$ is separable and metrizable. Now (c) follows from (b). 
Part (a) is presented in [20, p. 49] in case of the extended real line, and part (c) has been given by Tong [49] for the real line. There is another "classical" result on $\mathbb{R}$ concerning the existence of continuous functions which are in between a usc and an lsc function; see [49 and 11]. Our next result is an extension of this to a class of compact lattices.

The quoted results on $\mathbb{R}$ are at the roots of continuous selection theory. See the introductory section of [32]. In a sense, Michael focussed on the linear structure of $\mathbb{R}$, whereas we have been concentrating on the aspect of order.

6.6. Theorem. Let $L$ be a compact, connected, metrizable topological lattice with small meet-semilattices, let $Y$ be a $\mathrm{T}_{4}$ space, and let $f_{0}, f_{1}: Y \rightarrow L$ be such that $f_{0}$ is usc, $f_{1}$ is lsc, and $f_{0} \leq f_{1}$. Let $A \subseteq L$ be closed and let $f^{\prime}: A \rightarrow L$ be a continuous function such that $f_{0}\left|A \leq f^{\prime} \leq f_{1}\right| A$. Then there is a continuous function $f: Y \rightarrow L$ extending $f^{\prime}$, and such that $f_{0} \leq f \leq f_{1}$.

Note that if $A \subseteq Y$ is closed, then the function $f: Y \rightarrow[0,1]$, defined by $f(y)=0$ for $y \in A$ and $f(y)=1$ for $y \notin A$, is lsc and the function $1-f$ is usc. With these examples in mind, Theorem 6.6 can be seen to include the classical Urysohn and Tietze Theorems in topology.

Proof of the theorem. $L$ can be seen as a continuous (meet)-semilattice. Hence, its topology must be the "intrinsic" one. The fact that this topology also makes the "join"-operation continuous is a profitable bonus. The following multivalued function is LSC:

$$
F: Y \rightarrow L ; \quad F(y)=\left[f_{0}(y), f_{1}(y)\right] .
$$

Indeed, let $O \subseteq L$ be an open set meeting $F(y)$ in a point $u$. We assume that $O$ is of the "basic" type, $\gg(v) \cap \bigcap_{i=1}^{n} S \backslash \geq\left(v_{i}\right)$ (see 6.4 for notation). By the interpolation property, we can choose $v^{\prime}$ with $v \ll v^{\prime} \ll u$. Then $v^{\prime} \ll f_{1}(y)$, and since $f_{1}$ is lsc, there is a neighborhood $U_{1}$ of $y$ with

$$
\forall y^{\prime} \in U_{1}: \quad v^{\prime} \ll f_{1}\left(y^{\prime}\right) .
$$

We next show that for each $x \in L$ the function $Y \rightarrow L, y \mapsto f_{0}(y) \vee x$, is usc. To this end, suppose $f_{0}(y) \vee x \nsupseteq w$. The set $A_{w}=\{t \mid t \vee x \geq w\}$ is closed by the continuity of the operation " $\vee$ ". Also, $t \leq s$ and $t \in A_{w}$ imply $s \in A_{w}$; in particular, if $t \in A_{w}$ then $f_{0}(y) \nsupseteq t$. As $t$ is the supremum of all $t^{\prime} \ll t$, there is a $t^{\prime}$ of this kind with $f_{0}(y) \geq t^{\prime}$. As $f_{0}$ is usc, we obtain a neighborhood $U_{t}$ of $y$ with $f_{0}\left(y^{\prime}\right) \nsupseteq t^{\prime}$ for all $y^{\prime} \in U_{t}$. Now $\gg\left(t^{\prime}\right)$ is an open neighborhood of $t \in A_{w}$, and by the compactness of the latter, a finite number of sets $\gg\left(t^{\prime}\right)$ suffices to cover it. Suppose these sets correspond with $t_{1}, \ldots, t_{m}$. Then $U=\bigcap_{i=1}^{m} U_{t_{j}}$ is a neighborhood of $y$ with $f_{0}\left(y^{\prime}\right) \notin A_{w}$ for all $y^{\prime} \in U$.

Having established "usc", we proceed as follows. For each $i=1, \ldots, n$ we have $u \vee v^{\prime} \nsupseteq v_{i}$ and hence that $f_{0}(y) \vee v^{\prime} \nsupseteq v_{i}$. As we showed earlier, there is a neighborhood $U_{0}$ of $y$ with

$$
\forall y^{\prime} \in U_{0}: \quad f_{0}\left(y^{\prime}\right) \vee v^{\prime} \nsucceq v_{i}, \quad i=1, \ldots, n .
$$

By (1) and (2), for each $y^{\prime} \in U_{0} \cap U_{1}$,

$$
f_{0}\left(y^{\prime}\right) \leq f_{0}\left(y^{\prime}\right) \vee v^{\prime} \leq f_{1}\left(y^{\prime}\right) ; \quad v \ll f_{0}\left(y^{\prime}\right) \vee v^{\prime} \nsupseteq v_{i}, \quad i=1, \ldots, n,
$$

whence $F\left(y^{\prime}\right)$ meets $O$ in $f_{0}\left(y^{\prime}\right) \vee v^{\prime}$. 
The result now follows from the Selection Theorem.

The remainder of this section is concerned with fixed point problems. The first result does not involve the Selection Theorem, but relies on the auxiliary Theorem 4.1. Acyclicity is understood relative to Čech homology over a field.

6.7. Theorem. Let $X$ be a compact space, equipped with a closure stable and $\mathrm{S}_{4}$ convexity in which convex sets are connected. If $X$ is properly locally convex, then $X$ has the fixed point property for USC multifunctions with closed and acyclic value sets.

Proof. Let $C$ be a nonempty convex subset of $X$. If $\mathscr{U}$ is an open (in $X$ ) cover of $C$, then by proper local convexity there is a convex open cover of $C$ refining $\mathscr{U}$. The second cover has a contractible nerve on $C$. This shows that $C$ has trivial Cech homology, and it follows that $X$ is a convexoid space in the sense of Leray [31]. Alternatively, $X$ is an lc space (Begle, [3]).

We conclude that $X$ has a fixed point property as described above.

Aside of the "traditional" applications of this result, there is a new one on invariant arcs; see [62]. Keimel and Wieczorek [25] use the term Kakutani fixed point property to describe the condition that each USC multifunction with convex closed value sets, and mapping a topological convex structure $X$ into itself, has a fixed point. They obtained the remarkable result that if $X$ is compact and if each convex closed set has a base of convex closed neighborhoods, then $X$ has the Kakutani fixed point property provided all polytopes of $X$ with vertices in a prescribed dense subset of $X$ have the property.

Dugundji and Granas [15] observed that Michael's Selection Theorem leads to a fixed point theorem for LSC convex closed-value maps in a compact convex subset of locally convex vector space. A comparable result on compact trees (obtained with more elementary techniques) has appeared in [45].

We now use the quotient technique of 3.9 to obtain the following new result.

6.8. Theorem. Let $X$ be a compact space with a uniform $\mathrm{S}_{4}$ convexity in which convex sets are connected, and let $F: X \multimap X$ be an LSC multifunction with convex values. For each pseudo-metric $d$ on $X$ there is a point $x \in X$ with $d(x, F(x))=0$.

Proof. Let $q: X \rightarrow \widetilde{X}$ be the topological quotient corresponding to the pseudometric $d$. By the Factorization Theorem [39, 3.1], there is a uniform CP quotient $q^{*}: X \rightarrow X^{*}$ such that

(1) $q$ factors through $q^{*}$, that is: there is a map $g: X^{*} \rightarrow \tilde{X}$ such that $g \circ q^{*}=q$.

(2) The weight of $X^{*}$ equals the weight of $\tilde{X}$; in particular, $X^{*}$ is (topologically, hence also convexly) metrizable.

As we noted in 3.9, a uniform quotient maps convex sets to convex sets as well. This yields an LSC convex-valued multifunction $X \rightarrow X^{*} ; x \mapsto q^{*} F(x)^{-}$. By

(2) we can take a selection $f$. Now we have two maps $f, q^{*}: X \rightarrow X^{*}$, giving rise to a USC multifunction $X \rightarrow X, x \mapsto q^{*-1}(f(x))$ which by Theorem 6.7 has a fixed point $x$. By (1) we find that

$$
q(x)=g q^{*}(x)=g f(x) \in g q^{*} F(x)^{-}=q F(x),
$$

which is as desired. 
If the above quotient technique is applied to a compact convex subset $X$ of a locally convex vector space, then the resulting CP quotient $X^{*}$ need not be a standard convex set (see the example in 3.9). In this way, the above result really uses abstract convexity, even in standard situations, and one may wonder whether there is a proof of this result, which is exclusively in terms of standard convexity.

A second question is whether Theorem 6.8 can be strengthened to obtain a true fixed point theorem (at least, if the value sets are closed). Smithson's result in [45] shows it does for trees, but this case is perhaps not representative.

\section{REFERENCES}

1. S. M. Aseev, Approximation of semicontinuous multivalued mappings by continuous ones, Izv. Akad. Nauk SSSR Mat. 46 (1982), 460-476, 670. (Russian)

2. G. Beer, Approximate selections for upper semicontinuous convex valued multifunctions, $\mathrm{J}$. Approximation Theory 39 (1983), 172-184.

3. E. G. Begle, A fixed point theorem, Ann. of Math. (2) 51 (1950), 544-550.

4. C. Bessaga and A. Petczynski, Selected topics in infinite-dimensional topology, Monogr. Mat. 58, PWN-Polish Scientific Publishers, Warsaw, 1975, 353 pp.

5. K. Borsuk, Theory of retracts, PWN-Polish Scientific Publishers, Warsaw, 1967.

6. R. C. O'Brien, On the openness of the barycenter map, Math. Ann. 223 (1976), 207-212.

7. A. Cellina, $A$ theorem on the approximation of set-valued mappings, Atti Accad. Naz. Lincei Rend. Cl. Sci. Fis. Mat. Natur. (8) 47 (1969).

8. _ A further result on the approximation of set-valued mappings, Atti Accad. Naz. Lincei Rend. Cl. Sci. Fis. Mat. Natur. (8) 48 (1970).

9. H. Cohen, $A$ cohomological definition of dimension for locally compact Hausdorff spaces, Duke Math. J. 21 (1954), 209-224.

10. D. W. Curtis, Application of a selection theorem to hyperspace contractibility, Canad. J. Math. 37 (1985), 747-759.

11. C. H. Dowker, On countably paracompact spaces, Canad. J. Math. 3 (1951), 219-224.

12. P. Duchet, Convexity in combinatorial structures, Circ. Math. Palermo (2) Suppl. No 14 (1987), 261-293.

13. J. Dugundji, Absolute neighborhood retracts and local connectedness in arbitrary metric spaces, Compositio Math. 13 (1958), 229-246.

14. __ Topology, Allyn and Bacon, Boston, Mass., 1966.

15. J. Dugundji and A. Granas, Fixed point theory, PWN-Polish Scientific Publishers, Warsaw, 1982.

16. C. Eberhart, S. B. Nadler, Jr., and W. O. Nowell, Jr., Spaces of order arcs in hypperspaces, Fund. Math. 112 (1981), 111-120.

17. J. Eckhoff, Radon's theorem revisited, Contributions to Geometry, (J. Tölke and J. M. Wills, eds.), Proc. Geom. Sympos., Siegen, 1978, Birkhäuser, Basel, 1979, pp. 164-185.

18. J. W. Ellis, A general set-separation theorem, Duke Math. J. 19 (1952), 417-421.

19. R. Engelking, General topology, PWN-Polish Scientific Publishers, Warsaw, 1977, 626 pp.

20. G. Gierz, K. H. Hofmann, K. Keimel, J. D. Lawson, M. Mislove, and D. S. Scott, $A$ compendium of continuous lattices, Springer-Verlag, Berlin, $1980, \mathbf{x x}+371 \mathrm{pp}$.

21. R. E. Jamison, R. C. O'Brien, and P. D. Taylor, On embedding a compact convex set into a locally convex vector space, Pacific J. Math. 64 (1976), 193-205.

22. R. E. Jamison (R. E. Jamison-Waldner), A general theory of convexity, Dissertation, Univ. of Washington, Seattle, 1974.

23. _ Tietze's convexity theorem for semilattices and lattices, Semigroup Forum 15 (1978), 357-373. 
24. S. Kakutani, Ein Beweis des Sätzes von Edelheit über konvexe Mengen, Proc. Imp. Acad. Tokyo 13 (1937), 93-94.

25. K. Keimel and A. Wieczorek, Kakutani property of the polytopes implies Kakutani property of the whole space, Math. Ann. Appl. 130 (1988), 97-109.

26. J. L. Kelley and I. Namioka, Linear topological spaces, Graduate Texts in Math., no. 36, Springer-Verlag, Berlin, 1976.

27. G. Kozlowski, Images of ANR's, Trans. Amer. Math. Soc. (to appear).

28. G. Kozlowski, J. van Mill, and J. J. Walsh, AR-maps obtained from cell-like maps, Proc. Amer. Math. Soc. 82 (1981), 299-302.

29. J. D. Lawson, Intrinsic topologies in topological lattices and semilattices, Pacific J. Math. 44 (1973), 593-602.

30. _ Embeddings of compact convex sets and locally compact cones, Pacific J. Math. 66 (1976), 443-453.

31. J. Leray, Théorie des points fixes: indice total et nombre de Lefschetz, Bull. Soc. Math. France 87 (1959), 221-233.

32. E. Michael, Continuous selections. I, Ann. of Math. (2) 63 (1956), 361-382.

33. __ Continuous selections. II, Ann. of Math. (2) 64 (1956), 562-580.

34. __ Continuous selections. III, Ann. of Math. (2) 65 (1957), 357-390.

35. __ Convex structures and continuous selection, Canad. J. Math. 11 (1959), 556-575.

36. J. van Mill, Infinite-dimensional topology: Prerequisites and introduction, North-Holland, Amsterdam, 1988, $401 \mathrm{pp}$.

37. J. van Mill and M. van de Vel, Convexity preserving mappings in subbase convexity theory, Nederl. Akad. Wetensch. A 81 (1978), 76-90.

38. __ Subbases, convex sets, and hyperspaces, Pacific J. Math. 92 (1981), 385-402.

39. __ Equality of the Lebesgue and the inductive dimension functions for compact spaces with a uniform convexity, Colloq. Math. 50 (1986), 187-200.

40. S. B. Nadler, Jr., Hyperspaces of sets, Monographs in Pure and Appl. Math., 49, Dekker, New York, 1978, 707 pp.

41. L. Pasicki, On continuous selections, Opuscala Math. 3 (1988), 65-71.

42. A. Petrus, Contractibility of Whitney continua in $C(X)$, General Topology Appl. 9 (1978), 275-288.

43. C. P Pixley, An example concerning continuous selections on infinite-dimensional spaces, Proc. Amer. Math. Soc. 43 (1974), 237-244.

44. J. W. Roberts, The embedding of compact convex sets in locally convex spaces, Canad. J. Math. 30 (1978), 449-454.

45. R. E. Smithson, Fixed point theorems for certain classes of multifunctions, Proc. Amer. Math. Soc. 31 (1972), 595-600.

46. E. Spanier, Algebraic topology, McGraw-Hill, New York, 1966.

47. J. L. Taylor, A counterexample in shape theory, Bull. Amer. Math. Soc. 81 (1975), 629-632.

48. T. A. Tiller, Augmented compact spaces and continuous lattices, Houston J. Math. 7 (1981), 441-453.

49. H. Tong, Some characterizations of normal and perfectly normal spaces, Duke Math. J. 19 (1952), 289-292.

50. H. Torunczyk, On CE images of the Hilbert cube and characterization of Q-manifolds, Fund. Math. 106 (1980), 31-40.

51. M. van de Vel, Finite dimensional convex structures. I: general results, Topology Appl. 14 (1982), 201-225.

52. __ Euclidean convexity cannot be compactified, Math. Ann. 262 (1983), 563-572.

53. __ Pseudo-boundaries and pseudo-interiors for topological convexities, Dissertationes Math. 210 (1983), 1-72. 
54. __, Two-dimensional convexities are join-hull commutative, Topology Appl. 16 (1983), 181-206.

55. _ Binary convexities and distributive lattices, Proc. London Math. Soc. (3) 48 (1984), $1-33$.

56. __ Dimension of binary convex structures, Proc. London Math. Soc. (3) 48 (1984), 34-54.

57. _ Dimension of convex hyperspaces, Fund. Math. 122 (1984), 11-31.

58. __ Lattices and semilattices: a convex point of view, Continuous Lattices and their Applications (R.-E. Hoffmann \& K. H. Hofmann, eds.), Lecture Notes in Pure and Appl. Math., 101, Dekker, New York, 1984, pp. 279-302.

59. _ _ Convex Hilbert cubes in superextensions, Topology Appl. 22 (1986), 255-266.

60. _ Abstract, topological and uniform convex structures, Report WS-353, Monograph, Vrije Universiteit Amsterdam, 1989, 383 pp.

61. __ Collapsible polyhedra and median spaces, Report WS-360, 1990.

62. __ Invariant arcs, Whitney levels, and Kelley continua, Trans. Amer. Math. Soc. 326 (1991), 749-771.

63. A. Verbeek, Superextensions of topological spaces, Math. Centre Tracts, 41, Mathematisch Centrum, Amsterdam, 1972, 155 pp.

64. E. Verheul, Modular normed spaces (submitted).

65. L. E. Ward, Jr., A note on dendrites and trees, Proc. Amer. Math. Soc. 5 (1954), 992-994.

66. __ A note on Whitney maps, Canad. Math. Bull. 23 (1980), 373-374.

FAKUlteit Wiskunde en Informatika, VRIJe Universiteit, NL-1081 HV Amsterdam, The NETHERLANDS

E-mail address: marcel@cs.vu.nl 